\title{
DISSIPATION IN HAMILTONIAN SYSTEMS: DECAYING CNOIDAL WAVES*
}

\author{
G. DERKS $^{\dagger}$ AND E. VAN GROESEN $\ddagger$
}

\begin{abstract}
The uniformly damped Korteweg-de Vries (KdV) equation with periodic boundary conditions can be viewed as a Hamiltonian system with dissipation added. The KdV equation is the Hamiltonian part and it has a two-dimensional family of relative equilibria. These relative equilibria are space-periodic soliton-like waves, known as cnoidal waves.

Solutions of the dissipative system, starting near a cnoidal wave, are approximated with a long curve on the family of cnoidal waves. This approximation curve consists of a quasi-static succession of cnoidal waves. The approximation process is sharp in the sense that as a solution tends to zero as $t \rightarrow \infty$, the difference between the solution and the approximation tends to zero in a norm that sharply picks out their difference in shape. More explicitly, the difference in shape between a solution and a quasi-static cnoidal-wave approximation is of the order of the damping rate times the norm of the cnoidal-wave at each instant.
\end{abstract}

Key words. perturbed KdV equation, cnoidal waves, asymptotic behavior

AMS subject classifications. 35B20, 35Q53, 76B25

1. Introduction. Consider the uniformly damped one-dimensional Kortewegde Vries $(\mathrm{KdV})$ equation with periodic boundary conditions

$$
\begin{array}{ll}
u_{t}=-\partial_{x}\left[u_{x x}+u^{2}\right]-\varepsilon u, & t>0, \quad x \in(0,2 \pi), \\
u(0, t)=u(2 \pi, t), \quad u_{x}(0, t)=u_{x}(2 \pi, t), & t \geq 0 .
\end{array}
$$

In this equation $\varepsilon$ is a small parameter that gives the strength of the damping and the subscripts denote differentiation with respect to the given variable. Furthermore, we assume that the function $u(x, t)$ has mean value zero for all time:

$$
\int_{0}^{2 \pi} u(x, t) d x=0, \quad t \geq 0 .
$$

In [5] and [7], it is shown that the initial value problem of (1) with $\varepsilon=0$ is well posed in $H^{s}, s \geq 1$. It is easy to see that if $\varepsilon \neq 0$, this property remains; see, e.g., [14].

If $\varepsilon=0$, there is no damping present and the resulting equation is the $\mathrm{KdV}$ equation, which can be regarded as a Hamiltonian system with the Hamiltonian

$$
H(u)=\int_{0}^{2 \pi}\left[\frac{1}{2} u_{x}^{2}-\frac{1}{3} u^{3}\right] d x
$$

and with the operator $\partial_{x}$ as the structure map. The KdV equation was originally derived in 1895 as a model for planar, unidirectional waves propagating in shallow wa-

* Received by the editors August 6, 1993; accepted for publication (in revised form) March 13, 1995. This work is part of the research program of the Stichting voor Fundamenteel Onderzoek der Materie (FOM) and the Stichting Mathematisch Centrum (SMC), which are financially supported by the Nederlandse Organisatie voor Wetenschappelijk Onderzoek (NWO).

$\dagger$ Department of Applied Mathematics, University of Twente, P.O. Box 217, 7500 AE Enschede, The Netherlands. Current address: Department of Mathematical and Computing Sciences, University of Surrey, Guildford, Surrey GV2 5XH, United Kingdom.

$\ddagger$ Department of Applied Mathematics, University of Twente, P.O. Box 217, 7500 AE Enschede, The Netherlands. 
ter [21]. Over the last thirty years, the KdV equation has appeared as a model equation for many other physical situations that feature wave motion wherein nonlinearity and dispersion are comparable. For a review on the KdV equation, see [25] and [27].

The KdV equation is translation invariant. This invariance gives another first integral in the system besides the Hamiltonian, namely the $L^{2}$-norm of the solutions

$$
I(u)=\frac{1}{2} \int_{0}^{2 \pi} u^{2} d x
$$

(Moreover, the $\mathrm{KdV}$ equation is completely integrable, but here we use only the translation invariance.) The Hamiltonian $I$-flow is the translation operator (see [26])

$$
\left(\Phi_{\varphi}^{I}(u)\right)(x)=u(x+\varphi), \quad x \in[0,2 \pi], \varphi \in[0,2 \pi] .
$$

The tangent vector to this flow is the Hamiltonian $I$-vector field, denoted by

$$
X_{I}(u)=\partial_{x} I^{\prime}(u)=\partial_{x} u .
$$

Profiles of traveling-wave solutions of a translation-invariant Hamiltonian system can be found as critical points of the Hamiltonian for fixed values of $I$. In other words, they are relative equilibria (see [1]), and the family of all traveling-wave profiles is called the manifold of relative equilibria (MRE). In case of the periodic KdV equation, the relative equilibria are solitary-wave solutions, the so-called cnoidal waves. The cnoidal waves with minimal period $2 \pi$ form a two-dimensional family which can be parameterized with the value of the integral $I$ (a quantity related to the amplitude of the cnoidal wave) and the "position" of the cnoidal wave. The MRE consists of traveling-wave profiles, but for simplicity, the two-dimensional manifold consisting of the relative equilibrium solutions - hence the traveling-wave solutions - is also called the MRE. (Only when this can cause ambiguity, we will distinguish between these two manifolds by calling the second one the traveling-wave MRE.)

The cnoidal waves are orbitally stable solutions. In [4], this orbital stability is proved by using that in fact the cnoidal waves are constrained minima of the Hamiltonian for fixed values of the integral $I$. Here orbital stability means stability modulo translations. In other words, the profile of the cnoidal waves is dynamically stable; its "position" is ignored. This is the strongest kind of stability possible for this system because a small change in the speed or amplitude can cause a translational drift. For this reason, in this article, we consider only the profile of the waves and do not bother much about the "position" of the waves.

For the cnoidal waves, this implies that we are only interested in the one-dimensional family of wave profiles. For every fixed value of $I=\gamma$, we choose the cnoidal-wave profile that has its maximum at $x=0$ (this profile is symmetric around $x=0)$ and denote it by $\bar{u}(\gamma)$. Then the set

$$
\{\bar{u}(\gamma) \mid \gamma \geq 0\}
$$

is a one-dimensional submanifold of the MRE from which the translations are divided out. The wave speed of the cnoidal wave with $I=\gamma$ is denoted by $\lambda(\gamma)$; it is also the Lagrange multiplier in the Euler-Lagrange equation of the constrained critical-point problem

$$
\partial_{x}\left(\bar{u}_{x x}+\bar{u}^{2}+\lambda(\gamma) \bar{u}\right)=0 \quad \text { or } \quad \bar{u}_{x x}+\bar{u}^{2}+\lambda(\gamma) \bar{u}=\alpha(\gamma)
$$


with $\alpha(\gamma)=\frac{\gamma}{2 \pi}$, which follows by integrating the equation from 0 to $2 \pi$. The cnoidal waves can also be found as unconstrained critical points. To see this, for $\gamma \geq 0$, we define the modified KdV Hamiltonian

$$
H_{\gamma}(u)=H(u)-\lambda(\gamma) I(u)
$$

Then for every $\gamma \geq 0$, the cnoidal wave $\bar{u}(\gamma)$ is a critical point of $H_{\gamma}$.

The first general method for using the variational characterization of relative equilibria to draw conclusions about the stability was given in 1985 in [17]. Later, this method was extended to the energy-momentum method in [28] and [29]. In [15] and [23], the sufficient conditions for the stability of the relative equilibria were weakened. In this article, we will extend the use of the variational characterization of the relative equilibria to draw conclusions about the approximation with the cnoidal waves in the damped $\mathrm{KdV}$ equation.

For $\varepsilon \neq 0$, the cnoidal waves are no longer solutions of equation (1) and every solution decays to the zero state. This follows from the time behavior of the $L^{2}$-norm of $u$ (which equals $I(u)$ ):

$$
\frac{\mathrm{d}}{\mathrm{d} t} I(u)=\left(I^{\prime}(u), \partial_{x} H^{\prime}(u)-\varepsilon P(u)\right)=-\varepsilon \int_{0}^{2 \pi} u^{2} d x=-2 \varepsilon I(u) .
$$

(We use the notation $F^{\prime}(u)$ to denote the variational derivative of a differentiable functional $F(u)$.) In other words, (3) states that $I(u(t))=I(u(0)) e^{-2 \varepsilon t}$ and that $\lim _{t \rightarrow \infty} I(u(t))=0$, which implies that $\lim _{t \rightarrow \infty} u(t)=0$.

Although a solution never stays in the neighborhood of one specific cnoidal-wave profile, the full MRE can be useful to approximate the behavior of a solution that starts near a cnoidal wave. This behavior is indicated by numerical experiments and analytical approximations; see [16]. A similar behavior can be found (numerically and experimentally) for the $\mathrm{KdV}$ equation with dissipation on an infinite interval. However, in this case, some problems arise in the derivation of an analytical approximation since the decay of the mass functional $M(u)=\int u$ then has to be taken into account; see [18]-[20].

In this article, we approximate a solution of the damped $\mathrm{KdV}$ equation on a periodic interval by a projection of the solution on the MRE. An important issue in this article is the justification of the approximation of a solution with this projection. We will use a norm in the Sobolev space $H_{\mathrm{per}}^{1}$ to derive this justification. The usual $H_{\text {per }}^{1}$-norm is given by

$$
\|u\|_{H_{\mathrm{per}}^{1}}^{2}=\int_{0}^{2 \pi}\left[u(x)^{2}+u_{x}(x)^{2}\right] d x=\|u\|_{0}^{2}+\left\|u_{x}\right\|_{0}^{2}, \quad u \in H_{\mathrm{per}}^{1},
$$

where $\|\cdot\|_{0}$ denotes the $L_{\mathrm{per}}^{2}$-norm. Because we consider only functions with mean value zero, by the Poincaré inequality, the following norm is equivalent to the $H_{\text {per- }}^{1}$ norm:

$$
\|u\|_{1}^{2}=\int_{0}^{2 \pi} u_{x}(x)^{2} d x=\left\|u_{x}\right\|_{0}^{2}, \quad u \in H_{\mathrm{per}, 0}^{1}
$$

where $H_{\mathrm{per}, 0}^{1}$ is the subspace of $H_{\mathrm{per}}^{1}$ consisting of $2 \pi$-periodic functions with mean value 0 . Furthermore, we will often use the following Poincaré inequalities comparing 
the $L_{\mathrm{per}}^{2}$-norm (respectively, the $L_{\mathrm{per}}^{\infty}$-norm) and the $H_{\mathrm{per}, 0}^{1}$-norm:

$$
\begin{aligned}
\|u\|_{0}^{2} & =\int_{0}^{2 \pi}\left|\int_{x_{0}}^{x} u_{x}(\xi) d \xi\right|^{2} d x \leq \int_{0}^{2 \pi}\left[\int_{0}^{2 \pi}\left|u_{x}(\xi)\right| d \xi\right]^{2} d x \leq(2 \pi)^{2}\|u\|_{1}^{2}, \\
\|u\|_{\infty} & =\max _{x \in[0,2 \pi]}|u(x)|=\max _{x \in[0,2 \pi]}\left|\int_{x_{0}}^{x} u_{x}(\xi) d \xi\right| \leq \sqrt{ } 2 \pi\|u\|_{1} .
\end{aligned}
$$

Here $x_{0}$ denotes any zero of $u(t)$. (This zero exists because $u(t)$ has mean value zero.)

As we stated before, we are not interested in differences caused by translations. Therefore, we define (analogously to $[3,4]$ ) translation-invariant distances related to the $L_{\text {per- }}^{2}$ and $H_{\mathrm{per}, 0}^{1}$-norm, denoted by $\rho_{0}$ and $\rho_{1}$, respectively, as

$$
\rho_{i}\left(u_{1}, u_{2}\right)=\min _{\varphi \in[0,2 \pi]}\left\|\Phi_{\varphi}^{I}\left(u_{2}\right)-u_{1}\right\|_{i}=\min _{\varphi \in[0,2 \pi]}\left\|\Phi_{\varphi}^{I}\left(u_{1}\right)-u_{2}\right\|_{i}, \quad i=0,1 .
$$

See also Figure 1.
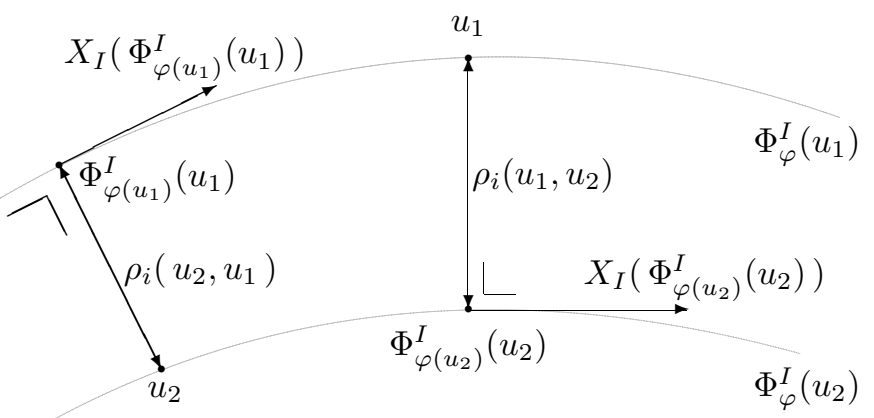

FIG. 1. The translation-invariant distance $\rho_{i}\left(u_{1}, u_{2}\right)=\rho_{i}\left(u_{2}, u_{1}\right)$. The translations $\Phi_{\varphi\left(u_{1}\right)}^{I}$ and $\Phi_{\varphi\left(u_{2}\right)}^{I}$ are such that $\rho_{i}\left(u_{1}, u_{2}\right)=\left\|\Phi_{\varphi\left(u_{1}\right)}^{I}\left(u_{1}\right)-u_{2}\right\|_{i}=\left\|\Phi_{\varphi\left(u_{2}\right)}^{I}\left(u_{2}\right)-u_{1}\right\|_{i}$.

To define a projection of a solution $u(t)$, we choose the wave profile on the MRE with an $I$-value equal to the $I$-value of the solution. Next, we define a position for this wave profile. It is obvious to choose the position such that the $\rho_{1}$-distance is as small as possible.

Definition 1.1. Let $u(t)$ be a solution of the damped KdV equation. Define the functions $\gamma(t) \in \mathbb{R}$ and $\varphi(t) \in \mathbb{R}$ such that

$$
\begin{aligned}
\gamma(t) & =I(u(t)), \\
\left\|\Phi_{-\varphi(t)}^{I}(u(t))-\bar{u}(\gamma)\right\|_{1} & =\min _{\varphi \in \mathbb{R}}\left\|\Phi_{\varphi}^{I}(u(t))-\bar{u}(\gamma)\right\|_{1}=\rho_{1}(\bar{u}(\gamma), u(t)) .
\end{aligned}
$$

Finally, we define $\xi(t)$ to be the difference between $u(t)$ and its projection

$$
\xi(t)=\Phi_{-\varphi(t)}^{I}(u(t))-\bar{u}(\gamma(t)) .
$$

The projection of the solution $t \rightarrow u(t)$ onto the MRE is the curve $t \rightarrow \Phi_{\varphi(t)}^{I}(\bar{u}(\gamma(t)))$. 
An important consequence of the choice of $\varphi$ as given by Definition 1.1 is that $\|\xi\|_{1}^{2}=\rho_{1}(\bar{u}(\gamma), u)$. Furthermore, the differential equation for the function $\gamma(t)$ is only in terms of $\gamma$ and hence can be solved explicitly (see equation (3)):

$$
\dot{\gamma}=-2 \varepsilon \gamma \quad \text { implying } \quad \gamma(t)=\gamma(0) e^{-2 \varepsilon t} .
$$

The main result of this article can now be formulated.

THEOREM 1.2. For every $\gamma_{0}>0$, there exists $a K>0$ and an $\varepsilon_{0}>0$ such that any solution $u(t)$ of the damped KdV equation (1) with $\varepsilon \leq \varepsilon_{0}$ that starts with $\gamma(0) \leq \gamma_{0}$ and, within a distance $\varepsilon$ of a cnoidal wave, stays in a relative $\varepsilon$-neighborhood of the family of cnoidal waves.

Explicitly, if $u(0)$ is such that $\gamma(0) \leq \gamma_{0}$ and $\rho_{1}(\bar{u}(\gamma(0)), u(0)) \leq \varepsilon$, then for all $t \geq 0$

$$
\rho_{1}(\bar{u}(\gamma(t)), u(t)) \leq K \varepsilon e^{-\varepsilon t}=\bar{K} \varepsilon\|\bar{u}(\gamma(t))\|_{0}=\tilde{K} \varepsilon\|\bar{u}(\gamma(t))\|_{1},
$$

where $\bar{K}=K / \sqrt{ } 2 \gamma(0)$.

In (6), we use that on every compact $\gamma$-interval $\|\bar{u}(\gamma)\|_{0}$ and $\|\bar{u}(\gamma)\|_{1}$ are of the same order. Hence on $\left[0, \gamma_{0}\right]$, the quotient $\|\bar{u}(\gamma)\|_{0} /\|\bar{u}(\gamma)\|_{1}$ can be estimated by a constant independent of $\gamma$.

Remark 1. Notice that the estimate for the initial condition and the estimate for the time behavior are in the same norm.

Remark 2. From Theorem 1.2, it can be deduced that for every $\gamma_{0}>0$, there exists a $\hat{K}$ and an $\hat{\varepsilon}_{0}$ such that if $H(u(0))-H(\bar{u}(\gamma(0))) \leq \hat{\varepsilon}_{0}$, then $H(u(t)) \leq \hat{K} I(u(t))$ for all $t$. This is sketched in Figure 2.

On the contrary, for the KdV-Burgers equation, i.e., $u_{t}=-\partial_{x}\left(u_{x x}+u^{2}\right)+\varepsilon u_{x x}$, we observe a "self-organization" towards the MRE. In an $H$ - $I$-figure, this means that every solution decays to zero tangent to the MRE. Hence asymptotically every solution will be below the tangent line to the $\mathrm{MRE}$ at 0 , hence below the line $H=I$. This behavior is sketched in Figure 3. This self-organization will not occur for the KdV equation with uniform damping. Hence the situation sketched in Figure 2 is also the best possible one. See [16] for more details.

Remark 3. In [14], the damped $\mathrm{KdV}$ equation with an additional forcing is considered and the existence of finite-dimensional attractors is investigated. In case there is no forcing, the attractor is trivial. The result in Theorem 1.2 gives more information than the existence of an attractor. It describes an approximation for the intermediate and asymptotic states. The asymptotic result also shows how the solution decays to the attractor 0 .

In the next sections, we will prove Theorem 1.2. The proof uses the variational principle which underlies the stability result of the cnoidal waves (see [4]). More explicit, to prove the stability of the cnoidal waves, we can use the (Lyapunov) functional $L(u)=H(u)-H(\bar{u}(I(u)))$. This functional is also similar to the so-called amended Hamiltonian or energy-momentum functional as used in [28, 29]. To prove Theorem 1.2, we will analyze the time behavior of the function $L(u(t))$, where $u(t)$ is a solution of the damped $\mathrm{KdV}$ equation, and derive a Gronwall-type inequality for $L(u(t))$. However, it turns out that this inequality is not optimal. To obtain optimal results in time asymptotics, a shift of the MRE to a neighboring $(\mathcal{O}(\varepsilon))$ set has to be performed. The use of such a shift can also be found in [22]. However, in that article, only equilibria of finite-dimensional perturbed Hamiltonian systems are investigated on a finite time scale. 


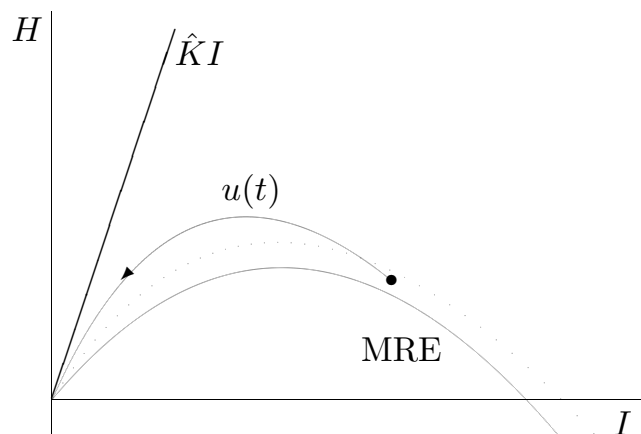

FIG. 2. Sketch of the projection of a solution of the uniformly damped KdV equation, which starts near the MRE, in the H-I-plane.

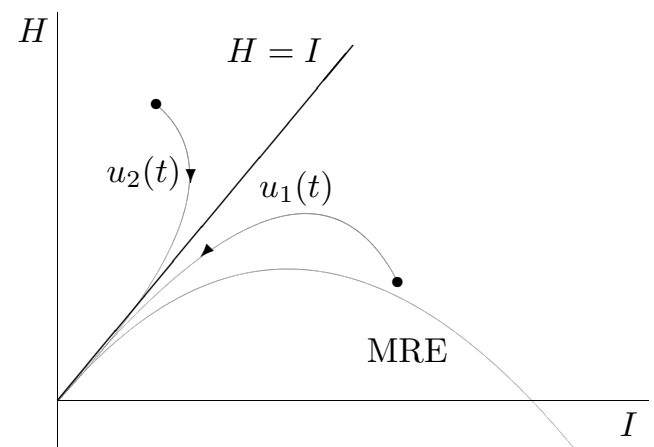

FIG. 3. Sketch of the projection of two solutions $u_{1}(t)$ and $u_{2}(t)$ of the $K d V$-Burgers equation in the H-I-plane. Note the tangent behavior near zero.

Remark 4. The shift of the MRE gives rise to an interesting question. The family of cnoidal waves is not invariant for the damped $\mathrm{KdV}$ equation. We will see that at every instance there is a forcing that drives a solution away from the MRE.

Keeping in mind the behavior as sketched in Figure 2, it is very unlikely that the MRE is stable for fixed values of $\varepsilon$. In other words, we cannot expect that for a fixed value of $\varepsilon$ it yields that for every $\delta>0$ there exist a $\delta_{0}>0$ and a $T>0$ such that for every solution $u(t)$ which satisfies $\rho_{1}(u(0), \bar{u}(\gamma(0)))<\delta_{0}$, it holds that $\rho_{1}(u(t), \bar{u}(\gamma(t)))<\delta$ for all $t \geq T$.

The question remains as to if there is another manifold near the MRE that is stable in this sense. A possible candidate could be the shift of the MRE, but we will see later that it has a disadvantage similar to that of the MRE, although it approximates the solution up to higher order in $\varepsilon$. However, that does not help for fixed values of $\varepsilon$. Another possibility could be an iterated shift of the MRE. If it exists, it would give an invariant manifold. However, this question regarding existence is not obvious to answer.

In [13], ideas similar to those used in this article are exploited to analyze the relevance of a two-dimensional family of relative equilibria of a finite-dimensional mechanical system with one cyclic coordinate to which uniform friction is added. An extension to higher-dimensional manifolds of relative equilibria of (finite-dimensional) Hamiltonian systems with symmetries and their relevance under a dissipative pertur- 
bation was recently established in [12].

2. A first analysis of the damped $\mathrm{KdV}$ equation. As seen in $\S 1$, the time behavior of the additional first integral $\gamma(t)=I(u(t))$, where $u(t)$ is a solution of the damped $\mathrm{KdV}$ equation, is given by

$$
\gamma(t)=I(u(t))=I(u(0)) e^{-2 \varepsilon t}=\gamma(0) e^{-2 \varepsilon t} .
$$

This implies that every solution converges to 0 and hence to the MRE. By Definition 1.1 it holds that $I(\bar{u}(\gamma)+\xi)=I(\bar{u}(\gamma))$, implying that

$$
-\int_{0}^{2 \pi} \bar{u} \xi=\frac{1}{2} \int_{0}^{2 \pi} \xi^{2}
$$

therefore,

$$
\|\xi(t)\|_{0}^{2}=2|(\bar{u}(\gamma(t)), \xi(t))| \leq 2\|\bar{u}(\gamma(t))\|_{0}\|\xi(t)\|_{0},
$$

and hence

$$
\|\xi(t)\|_{0} \leq 2 \sqrt{ } 2 \gamma(0) e^{-\varepsilon t}
$$

This implies that the translation-invariant $L^{2}$-distance between a solution and the MRE is less than or equal to a constant times the $L^{2}$-norm of the solution.

As we stated previously, to prove Theorem 1.2, we will make use of a similar (Lyapunov) functional as featured in the energy-momentum method to determine the stability of relative equilibria in an unperturbed Hamiltonian system (see [28, 29] or (for the KdV equation) [4]). To prove the stability of the cnoidal waves with such a technique, it is essential that the cnoidal wave $\bar{u}(\gamma)$ with minimal period $2 \pi$ is a constrained minimum of the Hamiltonian of the (unperturbed) KdV equation on the level set with $I=\gamma$. This property is proved in Lemma A.1 in the appendix. This lemma implies that the following functional acts as a Lyapunov functional for a cnoidal wave in the case of the unperturbed KdV equation.

Definition 2.1. Let $u \in H_{\mathrm{per}, 0}^{1}$. Define the functional $L(u)$ on $H_{\mathrm{per}, 0}^{1}$ as

$$
L(u)=H(u)-H(\bar{u}(\gamma))=H_{\gamma}(u)-H_{\gamma}(\bar{u}(\gamma))
$$

with $\gamma=I(u)$.

Furthermore, define the self-adjoint operator $\tilde{Q}(\gamma)$ on $H_{\text {per }}^{1}$ as

$$
\tilde{Q}(\gamma)=D^{2} \hat{L}(\bar{u}(\gamma))=D^{2} H_{\gamma}(\bar{u}(\gamma)) \mid=-D_{x x}-\lambda(\gamma)-2 \bar{u}(\gamma),
$$

and let $Q(\gamma)$ be its restriction on $H_{\mathrm{per}, 0}^{1}$; hence

$$
Q(\gamma)=\left.D^{2} \hat{L}(\bar{u}(\gamma))\right|_{H_{\mathrm{per}, 0}^{1}}=\left.D^{2} H_{\gamma}(\bar{u}(\gamma))\right|_{H_{\mathrm{per}, 0}^{1}} .
$$

The Euler-Lagrange equation (2), i.e., $\left(\bar{u}_{x x}+\bar{u}^{2}+\lambda \bar{u}\right)$ is constant, the notation of Definition 1.1, and identity (8) imply that $L(u)$ can be written as

$$
\begin{aligned}
L(u) & =H(\bar{u}+\xi)-H(\bar{u})=\int_{0}^{2 \pi}\left[\bar{u}_{x} \xi_{x}+\frac{1}{2} \xi_{x}^{2}-\bar{u}^{2} \xi-\bar{u} \xi^{2}-\frac{1}{3} \xi^{3}\right] \\
& =\int_{0}^{2 \pi} \xi\left[-\bar{u}_{x x}-\bar{u}^{2}-\lambda \bar{u}\right]+\int_{0}^{2 \pi}\left[\frac{1}{2} \xi_{x}^{2}-\bar{u} \xi^{2}+\lambda \bar{u} \xi-\frac{1}{3} \xi^{3}\right] \\
& =\int_{0}^{2 \pi}\left[\frac{1}{2} \xi_{x}^{2}-\bar{u} \xi^{2}-\frac{1}{2} \lambda \xi^{2}-\frac{1}{3} \xi^{3}\right]=\frac{1}{2}(Q(\gamma) \xi, \xi)-\frac{1}{3} \int_{0}^{2 \pi} \xi^{3}
\end{aligned}
$$


Define $\tilde{\mathbb{Y}}(\gamma)$ as the subspace of the tangent space to the $I$-level set that is orthogonal to the direction of the $I$-flow in $H_{\mathrm{per}}^{1}$, and define $\mathbb{Y}(\gamma)$ to be the restriction to $H_{\mathrm{per}, 0}^{1}$ :

$$
\begin{aligned}
& \tilde{\mathbb{Y}}(\gamma)=\left\{y \in H_{\mathrm{per}}^{1} \mid\left(y, X_{I}(\bar{u}(\gamma))\right)=0 \wedge\left(y, I^{\prime}(\bar{u}(\gamma))\right)=0\right\}, \\
& \mathbb{Y}(\gamma)=\left\{y \in H_{\mathrm{per}, 0}^{1} \mid\left(y, X_{I}(\bar{u}(\gamma))\right)=0 \wedge\left(y, I^{\prime}(\bar{u}(\gamma))\right)=0\right\} .
\end{aligned}
$$

From the minimality of the cnoidal waves, it follows that $Q(\gamma)$ is strictly positive definite on $\mathbb{Y}(\gamma)$ for a fixed value of $\gamma>0$.

For our purpose, we need a bit stronger property, namely that $L$ is equivalent with the translation-invariant $H_{\mathrm{per}}^{1}$-distance on every $L_{\mathrm{per}, 0}^{2}$-compact set, which includes 0 .

Lemma 2.2. For every compact interval $\mathcal{G} \in \mathbb{R}_{0}^{+}$with $0 \in \mathcal{G}$, there exist $C \geq c>0$ and a neighborhood $\mathcal{U} \subset H_{\mathrm{per}, 0}^{1}$ of the $M R E$ such that for all $u \in \mathcal{U}$ with $I(u) \in \mathcal{G}$, it holds that

$$
c \rho_{1}^{2}(\bar{u}(\gamma), u) \leq L(u) \leq C \rho_{1}^{2}(\bar{u}(\gamma), u)
$$

with $\gamma=I(u)$.

Proof. Let $\gamma>0$. First, we prove that $L$ is bounded from above. Let $u \in H_{\mathrm{per}, 0}^{1}$ and write $u=\Phi_{\varphi}^{I}(\bar{u}(\gamma)+\xi)$ as in Definition 1.1. Using the Poincarè inequalities (4) and (5), it is easy to calculate that there exists some $C_{1}(\gamma)>0$ such that

$$
\begin{aligned}
L(u) & =\int_{0}^{2 \pi}\left[\frac{1}{2} \xi_{x}^{2}-\bar{u} \xi^{2}-\frac{1}{2} \lambda \xi^{2}-\frac{1}{3} \xi^{3}\right] \\
& \leq \frac{1}{2}\|\xi\|_{1}^{2}+\|\bar{u}\|_{\infty}\|\xi\|_{0}^{2}+\frac{1}{2}|\lambda|\|\xi\|_{0}^{2}+\frac{1}{3}\|\xi\|_{\infty}\|\xi\|_{0}^{2} \\
& \leq 2 \pi^{2}\|\xi\|_{1}^{2}\left[\frac{1}{4 \pi^{2}}+2\|\bar{u}\|_{\infty}+|\lambda|+\frac{2 \sqrt{ } 2 \pi}{3}\|\xi\|_{1}\right] \\
& \leq C_{1}(\gamma)\|\xi\|_{1}^{2}
\end{aligned}
$$

in a neighborhood of the MRE, e.g., if $\|\xi\|_{1} \leq 1$. By definition, $\|\xi\|_{1}^{2}=\rho_{1}(u, \bar{u})$; hence equation (11) implies that

$$
L(u) \leq C_{1}(\gamma) \rho_{1}^{2}(u, \bar{u}(\gamma))
$$

and $C_{1}(\gamma)$ is bounded if $\gamma \rightarrow 0$.

Lemma A.3 in the appendix yields that $Q(\gamma)$ is strictly positive definite on $\mathbb{Y}(\gamma)$; explicitly,

$$
(Q(\gamma) y, y) \geq c_{1}(\gamma)\|y\|_{1}^{2} \quad \text { for all } y \in \mathbb{Y}(\gamma)
$$

with $c_{1}(\gamma)>0$ and $\lim _{\gamma \rightarrow 0} c_{1}(\gamma)>0$ as well.

This inequality implies a lower bound on $L$. To see this, write $\xi=a X_{I}(\bar{u})+$ $b I^{\prime}(\bar{u})+y$, where $y \in \mathbb{Y}(\gamma)$. We will show that $a$ and $b$ are of the order $\|\xi\|_{1}^{2}$ if $\|\xi\|_{1}$ is small. First, we estimate $a$. We know that $\left(\xi, X_{I}\left(\Phi_{\varphi(u)}^{I}(u)\right)\right)=0$; therefore,

$$
\begin{aligned}
0 & =\left(\xi, X_{I}\left(\Phi_{\varphi(u)}^{I}(u)\right)\right)=\left(\xi, X_{I}(\bar{u})\right)+\mathcal{O}\left(\|\xi\|_{0}^{2}\right) \\
& =a\left\|X_{I}(\bar{u})\right\|_{0}^{2}+\mathcal{O}\left(\|\xi\|_{1}^{2}\right) .
\end{aligned}
$$

Next we estimate $b$, using the fact that $I\left(\Phi_{\varphi(u)}^{I}(u)\right)=I(u)=I(\bar{u})$, by

$$
\begin{aligned}
0 & =I\left(\Phi_{\varphi(u)}^{I}(u)\right)-I(\bar{u})=\left(I^{\prime}(\bar{u}), \xi\right)+\mathcal{O}\left(\|\xi\|_{0}^{2}\right) \\
& =b\left\|I^{\prime}(\bar{u})\right\|_{0}^{2}+\mathcal{O}\left(\|\xi\|_{1}^{2}\right) .
\end{aligned}
$$


Now we know that the "largest" part of $\xi$ is in $\mathbb{Y}(\gamma)$, and we can derive a relation between $L(u)$ and $\|\xi\|_{1}^{2}$ for $\|\xi\|_{1}$ small:

$$
\begin{aligned}
L(u) & =L\left(\Phi_{\varphi(u)}^{I}(u)\right)=L(\bar{u})+\frac{1}{2}(Q(\gamma) \xi, \xi)+\mathcal{O}\left(\|\xi\|_{0}^{3}\right) \\
& =\frac{1}{2}(Q(\gamma) y, y)+\mathcal{O}\left(|b|\|\xi\|_{0}+|b|^{2}+\|\xi\|_{0}^{3}\right) \\
& \geq \frac{1}{2} c_{1}(\gamma)\|y\|_{1}^{2}+\mathcal{O}\left(\|\xi\|_{0}^{3}\right)=\frac{1}{2} c_{1}(\gamma)\|\xi\|_{1}^{2}+\mathcal{O}\left(\|\xi\|_{0}^{3}\right) .
\end{aligned}
$$

This means that there exists a $\delta_{0}>0$ such that for all $u$ with $\rho_{1}(\bar{u}, u)<\delta_{0}$ (recall that $\left.\|\xi\|_{1}^{2}=\rho_{1}(u, \bar{u})\right)$,

$$
L(u) \geq \frac{1}{4} c_{1}(\gamma) \rho_{1}(\bar{u}, u) .
$$

To prove equation (10), we use the facts that $\lim _{\gamma \rightarrow 0} c_{1}(\gamma) \neq 0$ and $C_{1}(\gamma)$ is bounded. Hence in every compact interval $\mathcal{G}$ that includes 0 , there exist $0<c \leq C$ such that (10) holds.

After these observations about the unperturbed KdV equation, we return to the damped $\mathrm{KdV}$ equation. The time behavior of the difference function $\xi(t)$ gives an idea about what causes the deviation of solutions of the damped KdV equation of the MRE. Using Definition 1.1, we see that

$$
\begin{aligned}
\dot{\xi} & =\frac{\mathrm{d}}{\mathrm{d} t}\left[\Phi_{-\varphi(t)}^{I}(u(t))\right]-\dot{\bar{u}}(\gamma(t)) \\
& =-\partial_{x}\left[\bar{u}_{x x}+\xi_{x x}+2(\bar{u}+\xi)^{2}\right]-\varepsilon(\bar{u}+\xi)-\dot{\bar{u}}-\dot{\varphi} \partial_{x}(\bar{u}+\xi) .
\end{aligned}
$$

To recognize more structure in this equation, we will rewrite it. By using the EulerLagrange equation (27) (in the appendix) and the differential equation for $\gamma$, it follows that

$$
\begin{aligned}
\dot{\xi} & =\partial_{x}\left[\xi_{x x}+2 \bar{u} \xi+\lambda \xi+\xi^{2}\right]+(\lambda-\dot{\varphi}) \partial_{x}(\bar{u}+\xi)-\varepsilon\left[2 \gamma \bar{u}^{\prime}(\gamma)-\bar{u}\right]-\varepsilon \xi \\
& =\partial_{x} H_{\gamma}{ }^{\prime}(\bar{u}+\xi)+(\lambda-\dot{\varphi}) \partial_{x}(\bar{u}+\xi)-\varepsilon \xi+R(\bar{u}(\gamma), \varepsilon)
\end{aligned}
$$

with the so-called residual

$$
R(\bar{u}(\gamma), \varepsilon)=-\varepsilon\left[2 \gamma \bar{u}^{\prime}(\gamma)-\bar{u}\right]
$$

The first two terms of the equation for $\dot{\xi}$ have a Hamiltonian origin. The first term is the modified KdV Hamiltonian. The second term induces a translation of the wave profile; hence this term will be irrelevant for our analysis. The third and fourth terms are the most relevant for our analysis. The third term represents the damping. The fourth term does not depend on $\xi$. It is called the residual because it shows the effect of the damped $\mathrm{KdV}$ equation on the MRE, except for some irrelevant influence in the translation direction $X_{I}(\bar{u})$. The residual is an element of $\mathbb{Y}(\gamma)$; hence if the residual is not equal to 0 , then a solution that starts on the MRE will soon deviate from it. This implies that the residual acts like a forcing in the $\xi$-equation. In other words, the third and fourth terms show a competition between a dissipation directed towards the MRE and a forcing away from the MRE.

We have seen that the functional $L$ is equivalent to the $H_{1}$-norm of $\xi$. Therefore, we look at the time behavior of this functional to see how fast $\xi$ can grow. Using the 
translation invariance of $H$ and $I$, it follows immediately that $L(u)=L(\bar{u}+\xi)$. Using the second expression in (9) and the equation for $\dot{\xi}$, it follows that

$$
\frac{\mathrm{d}}{\mathrm{d} t} L(u(t))=\dot{\lambda}[I(\bar{u}+\xi)-I(\bar{u})]+\left(H_{\gamma}{ }^{\prime}(\bar{u}+\xi),-\varepsilon \xi+R(\bar{u}, \varepsilon)-\dot{\bar{u}}\right) .
$$

The first term is zero because $I(\bar{u}+\xi)=I(\bar{u})$. Note that $H_{\gamma}^{\prime}(\bar{u}+\xi)=Q(\gamma) \xi-\xi^{2}$; hence $\left(H_{\gamma}{ }^{\prime}(\bar{u}+\xi),-\varepsilon \xi\right)=-2 \varepsilon L(u)+{ }_{3}^{1}\left(\xi^{2}, \xi\right)$. We again use the Euler-Lagrange equation to rewrite the inner product with $(R(\bar{u}, \varepsilon)-\dot{\bar{u}})$. This yields

$$
\frac{\mathrm{d}}{\mathrm{d} t} L(u(t))=-2 \varepsilon L(\gamma, \xi)+\varepsilon \int_{0}^{2 \pi} \bar{u}^{2} \xi+\varepsilon \int_{0}^{2 \pi} \bar{u} \xi^{2}+\frac{1}{3} \varepsilon \int_{0}^{2 \pi} \xi^{3} .
$$

Note that in expression (14), for the time behavior of $L$, the first term is dissipative, the second is a forcing-like component, and the last two terms are small compared to the first two terms (if $\xi$ and $\gamma$ are small).

With this expression for $\underset{\mathrm{d} t}{\mathrm{~d}} L(u(t))$, we derive a preliminary estimate for the functional $L$.

Proposition 2.3. For every $\varepsilon>0$, there exist $a \delta>0$ and a constant $K_{0}$, both depending on $\gamma(0)$, such that

$$
\sqrt{ } L(u(t)) \leq K_{0}[\sqrt{ } L(u(0))+2 \sqrt{ } 2 \pi \gamma(0)] e^{-\varepsilon t}
$$

as long as $\|\xi(t)\|_{1}<\delta$.

Proof. For the last three terms in equation (14), it holds that

$$
\begin{aligned}
\left|\int_{0}^{2 \pi} \bar{u}^{2} \xi\right| & \leq\|\xi\|_{\infty} \int_{0}^{2 \pi} \bar{u}^{2} \leq 2 \sqrt{ } 2 \pi \gamma\|\xi\|_{1}, \\
\left|\int_{0}^{2 \pi} \bar{u} \xi^{2}\right| & \leq\|\xi\|_{\infty} \int_{0}^{2 \pi} \xi \bar{u} \leq \sqrt{ } 2 \pi\|\xi\|_{1}\|\bar{u}\|_{0}\|\xi\|_{0} \leq 4 \pi \sqrt{ } \pi \sqrt{ } \gamma\|\xi\|_{1}^{2}, \\
\left|\int_{0}^{2 \pi} \xi^{3}\right| & \leq\|\xi\|_{\infty} \int_{0}^{2 \pi} \xi^{2} \leq \sqrt{ } 2 \pi\|\xi\|_{1}\|\xi\|_{0}^{2} \leq 8 \pi \sqrt{ } \pi \sqrt{ } \gamma\|\xi\|_{1}^{2} .
\end{aligned}
$$

In these estimates, we use that $\|\xi\|_{0} \leq 2 \sqrt{ } 2 \gamma$ and the Poincaré inequalities (4) and (5).

To be able to switch from $\|\xi\|_{1}$ to the functional $L$, we will use Lemma 2.2. Let $\delta$ be such that the equivalence relation (10) holds for all $\|\xi\|_{1}<\delta$. Substituting the relations above into (14) and using (10) gives the following estimate for the time behavior of $L$ :

$$
\frac{\mathrm{d}}{\mathrm{d} t} L \leq\left[-2 \varepsilon+\varepsilon \frac{20 \sqrt{ } \gamma \pi \sqrt{ } \pi}{3 c}\right] L+\varepsilon \frac{2 \sqrt{ } 2 \pi \gamma}{\sqrt{ } c} \sqrt{ } L .
$$

Define $N(t)=e^{\varepsilon t} \sqrt{ } L$; then this inequality implies that $N(t)=0$ or

$$
\dot{N} \leq\left[\begin{array}{c}
10 \sqrt{ } \gamma \pi \sqrt{ } \pi \\
3 c
\end{array}\right] N+\varepsilon \frac{\sqrt{ } 2 \pi \gamma}{\sqrt{ } c} e^{\varepsilon t}
$$

Applying Gronwall's lemma to this equation gives

$$
N(t) \leq K_{0} N(0)+\varepsilon K_{0} \int_{0}^{t} \frac{\sqrt{ } 2 \pi e^{-\varepsilon \tau} \gamma(0)}{\sqrt{ } c} d \tau=K_{0}\left[N(0)+\frac{\sqrt{ } 2 \pi \gamma(0)}{\sqrt{ } c}\left(1-e^{-\varepsilon t}\right)\right],
$$


where $K_{0}=\exp \left[\frac{10 \pi \sqrt{ } \pi \sqrt{ } \gamma(0)}{3 c}\right]$.

The estimate of Proposition 2.3 only provides information about the approximation on a finite time scale. Even if we start on the MRE, hence with $\|\xi(0)\|_{1}=0$, after some time the norm of the right-hand side of estimate (15) is of order $\sqrt{ } \gamma$ instead of order $\varepsilon \sqrt{ } \gamma$. This effect is induced by the residual, which after an integration becomes of order 1 instead of order $\varepsilon$. In other words, we need a smaller residual. The present residual is induced by the projection on the MRE, which approximates the solution in zeroth order. If we have a better approximation than this projection, we can expect a smaller residual. In the next section, we will derive such a better approximation and prove Theorem 1.2 by using a functional related to this better approximation.

3. Justification of the approximation. The residual $R(\bar{u}(\gamma), \varepsilon)$ measures how well the curve $\bar{u}(\gamma(t))$ obeys the damped KdV equation. The function $t \rightarrow \bar{u}(\gamma(t))$ is a zeroth-order approximation of the damped $\mathrm{KdV}$ equation and therefore gives a residual of $\operatorname{order} \varepsilon$. It can be expected that the residual for a first-order approximation of the damped $\mathrm{KdV}$ equation is smaller, of order $\varepsilon^{2}$. Using the knowledge that the $L^{2}$-norm $I(u)$ is $\gamma(0) e^{-2 \varepsilon t}$, which is a slow time behavior, we try to find a better approximation of the form

$$
u(t)=\Phi_{\lambda(\varepsilon) t}^{I}\left(\bar{u}(\gamma(t))+\varepsilon \bar{v}_{1}(\gamma(t), \varepsilon)\right)
$$

Substitution of this expression in the dynamical system (1) gives

$$
\partial_{x}\left[H^{\prime}\left(\bar{u}+\varepsilon \bar{v}_{1}\right)-\lambda(\varepsilon) I^{\prime}\left(\bar{u}+\varepsilon \bar{v}_{1}\right)\right]-\varepsilon\left[\left(\bar{u}+\varepsilon \bar{v}_{1}\right)-2 \gamma\left(\bar{u}^{\prime}(\gamma)+\bar{v}_{1}^{\prime}(\gamma, \varepsilon)\right)\right]=0 .
$$

After taking first-order terms in $\varepsilon$ of this equation, it remains (up to order- $\varepsilon^{2}$ terms)

$$
H^{\prime}\left(\bar{u}+\varepsilon \bar{v}_{1}\right)-\lambda(\varepsilon) I^{\prime}\left(\bar{u}+\varepsilon \bar{v}_{1}\right)=\partial_{x}^{-1}[R(\bar{u}, \varepsilon)]+\alpha(\varepsilon) \text {. }
$$

(The operator $\partial_{x}^{-1}$ is defined to act on the space $H_{\mathrm{per}, 0}^{1}$ and $\alpha(\varepsilon)$ is a constant which is introduced by the integration.) If we can find a solution $\left(\bar{u}_{1}(\gamma, \varepsilon), \lambda(\varepsilon), \alpha(\varepsilon)\right)$ of equation (16) (with $\bar{u}_{1}(\gamma, \varepsilon)=\bar{u}(\gamma)+\varepsilon \bar{v}_{1}(\gamma, \varepsilon)$ ), then we expect that the residual in $\bar{u}_{1}(\gamma, \varepsilon)$ is of order $\varepsilon^{2}$, an improvement compared to the residual in $\bar{u}(\gamma)$, which is of order $\varepsilon$.

Another way to interpret the definition of the function $\bar{u}_{1}(\gamma, \varepsilon)$ is by noticing that $\bar{u}_{1}(\gamma, \varepsilon)$ is a constrained critical point of a new Hamiltonian

$$
H_{\text {new }}(u, \gamma, \varepsilon)=H(u)-\left(\partial_{x}^{-1} R(\bar{u}(\gamma), \varepsilon), u\right)
$$

on the level set of $I(u)=\gamma$. Hence $\bar{u}_{1}$ is a kind of new relative equilibrium. However, the new Hamiltonian $H_{\text {new }}$ is not translation invariant; hence neither can we find a two-parameter family of constrained critical points nor is $\Phi_{\lambda(\varepsilon) t}^{I}\left(\bar{u}_{1}\right)$ a solution of the new Hamiltonian system. Because we ignore all shifts in the solution, a curve of new relative equilibria $\bar{u}_{1}(\gamma, \varepsilon)$ is sufficient to give a better approximation for a solution of the damped KdV equation.

Remark 5. It is possible to define a new translation-invariant Hamiltonian which possesses a two-dimensional family of relative equilibria that give a residual of order $\varepsilon^{2}$. Analogously to the definition of the translation-invariant distance, we define this new Hamiltonian as

$$
\tilde{H}_{\text {new }}(u, \gamma, \varepsilon)=H(u)-\left(\partial_{x}^{-1} R(\bar{u}, \varepsilon), \Phi_{\psi(u)}^{I}(u)\right)
$$


with the functional $\psi: H_{\text {per }, 0}^{1} \rightarrow[0,2 \pi)$ such that

$$
\left(\partial_{x}^{-1} R(\bar{u}, \varepsilon), \Phi_{\psi(u)}^{I}(u)\right)=\min _{\varphi \in[0,2 \pi)}\left(\partial_{x}^{-1} R(\bar{u}, \varepsilon), \Phi_{\varphi}^{I}(u)\right) .
$$

If the minimum in (17) is attained at more than one value $\varphi \in[0,2 \pi)$, then $\psi(u)$ is the smallest one. Now $H_{\text {new }}$ is a translation-invariant functional and its derivative with respect to $u$ is

$$
\tilde{H}_{\text {new }}^{\prime}(u, \gamma, \varepsilon)=H^{\prime}(u)-\Phi_{-\psi(u)}^{I}\left(\partial_{x}^{-1} R(\bar{u}, \varepsilon)\right) .
$$

Hence the constrained critical points $\tilde{u}_{1}$ of $\tilde{H}_{\text {new }}$ on the level set $I=\gamma$ satisfy

$$
0=H^{\prime}\left(\tilde{u}_{1}\right)-\tilde{\lambda}_{1} I^{\prime}\left(\tilde{u}_{1}\right)-\Phi_{-\psi(u)}^{I}\left(\partial_{x}^{-1} R(\bar{u}, \varepsilon)\right) .
$$

Because $\psi(\bar{u})=0$, the approximation $\bar{u}_{1}$ gives rise to a residual of order $\varepsilon^{2}$. See [10] for more details.

First, we show that there indeed exists a curve of new relative equilibria $\bar{u}_{1}(\gamma, \varepsilon)$ in the neighborhood of $\varepsilon=0$ by applying the implicit-function theorem to $H_{\text {new }}$, which is a perturbation of the original Hamiltonian $H$. To be able to apply the implicit-function theorem, it is important that $\partial_{x}^{-1} R(\bar{u}(\gamma), \varepsilon)$ is orthogonal to the kernel of $Q(\gamma)$ and hence orthogonal to $X_{I}(\bar{u})$. This will be shown in the proof of the next lemma. By taking the inner product with $X_{I}\left(\bar{u}_{1}\right)$ in equation (16), it follows that $\bar{u}_{1}$ is orthogonal to $R(\bar{u}(\gamma), \varepsilon)$.

Lemma 3.1. For every $\gamma>0$ there exists an $\varepsilon_{0}(\gamma)$ and a unique curve

$$
\left\{\bar{u}_{1}(\gamma, \varepsilon)|| \varepsilon \mid \leq \varepsilon_{0}(\gamma)\right\}
$$

of minimal points of the Hamiltonian $H_{\text {new }}$ on the level set $I=\gamma$ in $H_{\mathrm{per}, 0}^{1}$. Explicitly, for every $|\varepsilon| \leq \varepsilon_{0}(\gamma)$, there exist unique Lagrange multipliers $\lambda_{1}(\gamma, \varepsilon)$ and $\alpha_{1}(\gamma, \varepsilon)$ such that

$$
\begin{aligned}
& 0=H_{\text {new }}^{\prime}\left(\bar{u}_{1}(\gamma, \varepsilon), \gamma, \varepsilon\right)-\lambda_{1}(\gamma, \varepsilon) I^{\prime}\left(\bar{u}_{1}(\gamma, \varepsilon)\right)-\alpha_{1}(\gamma, \varepsilon) \\
& 0=I\left(\bar{u}_{1}(\gamma, \varepsilon)\right)-\gamma
\end{aligned}
$$

Furthermore, there exists a $K(\gamma)>0$ such that for all $|\varepsilon| \leq \varepsilon_{0}(\gamma)$, it holds that

$$
\begin{aligned}
\left\|\bar{u}(\gamma)-\bar{u}_{1}(\gamma, \varepsilon)\right\|_{1} & \leq K(\gamma)\left\|\partial_{x}^{-1} R(\bar{u}(\gamma), \varepsilon)\right\|_{0}=\mathcal{O}\left(\varepsilon\|\bar{u}(\gamma)\|_{0}\right), \\
\left|\lambda(\gamma)-\lambda_{1}(\gamma, \varepsilon)\right| & \leq K(\gamma)\left\|\partial_{x}^{-1} R(\bar{u}(\gamma), \varepsilon)\right\|_{0}=\mathcal{O}\left(\varepsilon\|\bar{u}(\gamma)\|_{0}\right) .
\end{aligned}
$$

Finally, $\lim _{\gamma \rightarrow 0} K(\gamma)$ and $\lim _{\gamma \rightarrow 0} \varepsilon_{0}(\gamma)$ exist and $\varepsilon_{0}(0)>0$.

Proof. Let $\gamma>0$. As we stated previously, we use the implicit-function theorem (see, e.g., [9]) to prove this lemma. First, we reformulate the problem. Instead of looking for a $2 \pi$-periodic solution of (18) with mean value, it is more convenient to add the mean-value zero condition to the equations and consider the problem in the space of all $2 \pi$-periodic functions. Hence we look for a $2 \pi$-periodic solution of

$$
0=\left(\begin{array}{c}
H^{\prime}(u)-\lambda I^{\prime}(u)-\alpha \mathbf{1} \\
I(u)-\gamma \\
M(u)
\end{array}\right)-\left(\begin{array}{c}
\partial_{x}^{-1} R(\bar{u}(\gamma), \varepsilon) \\
0 \\
0
\end{array}\right),
$$

where 1 is the function that equals 1 for all $x \in[0,2 \pi]$ and $M(u)=\int_{0}^{2 \pi} u$. For $\varepsilon=0$ (the unperturbed case), this problem does not have a unique solution in $H_{\mathrm{per}}^{1}$. We 
have seen that a one-dimensional manifold of solutions can be formed: all translates of the cnoidal wave $\bar{u}(\gamma)$, hence $\left\{\left(\Phi_{\varphi}^{I}(\bar{u}(\gamma)), \lambda(\gamma), \alpha(\gamma)\right) \mid \varphi \in[0,2 \pi)\right\}$. Hence this will cause a problem in the application of the implicit-function theorem to these equations in $H_{\text {per }}^{1}$. To avoid this problem, we will use the fact that $\bar{u}_{1}$ is orthogonal to $R(\bar{u}, \varepsilon)$.

We distinguish two cases.

1. If $\left.\left(R(\bar{u}, \varepsilon), \bar{u}_{x}\right)\right) \neq 0$, then we add the equation

$$
0=\left(\partial_{x}^{-1} R(\bar{u}, \varepsilon), \partial_{x} u\right)=-(R(\bar{u}, \varepsilon), u)
$$

to our set of equations (19), and we add the term $\beta X_{I}(u)$ with the extra unknown $\beta$ to the first equation. Explicitly, for $\varepsilon \geq 0$, we introduce the functions $F$ and $F_{0}$ on $H_{\text {per }}^{1} \times \mathbb{R} \times \mathbb{R} \times \mathbb{R}:$

$$
F(u, \lambda, \alpha, \beta ; \varepsilon)=\left(\begin{array}{c}
H^{\prime}(u)-\lambda I^{\prime}(u)-\alpha \mathbf{1}-\beta X_{I}(u)-\partial_{x}^{-1} R(\bar{u}(\gamma), \varepsilon) \\
I(u)-\gamma \\
M(u) \\
(R(\bar{u}, \varepsilon), u)
\end{array}\right)
$$

and $F_{0}(u, \lambda, \alpha, \beta)=F(u, \lambda, \alpha, \beta ; 0)$ for all $u \in H_{\mathrm{per}}^{1}$ and $\lambda, \alpha, \beta \in \mathbb{R}$. A solution of the equation

$$
F(u, \lambda, \alpha, \beta ; \varepsilon)=0
$$

gives a constrained critical point of the new Hamiltonian $H_{\text {new }}$ on level sets of $I$. Indeed, take the inner product of $X_{I}(u)$ with the first equation in $F=0$; then it follows that $\beta=0$. In other words, the first equation in $F=0$ is the Euler-Lagrange equation for the critical-point problem. Furthermore, if $\varepsilon=0$, the critical-point problem for the $\mathrm{KdV}$ equation reappears; hence $F_{0}(\bar{u}(\gamma), \lambda(\gamma), \alpha(\gamma), 0)=0$ and this solution is unique for $\varepsilon=0$ in $H_{\mathrm{per}}^{1} \times \mathbb{R} \times \mathbb{R} \times \mathbb{R}$ thanks to the last equation.

The function $F_{0}$ satisfies the following properties:

(i) $F_{0}$ is continuously differentiable. Indeed, it is a straightforward calculation to see that for all $(u, \lambda, \alpha, \beta),(\hat{u}, \hat{\lambda}, \hat{\alpha}, \hat{\beta}) \in H_{\text {per }}^{1} \times \mathbb{R} \times \mathbb{R} \times \mathbb{R}$,

$$
F_{0}(u, \lambda, \alpha, \beta)=F_{0}(\hat{u}, \hat{\lambda}, \hat{\alpha}, \hat{\beta})+D F_{0}(\hat{u}, \hat{\lambda}, \hat{\alpha}, \hat{\beta})\left(\begin{array}{c}
u-\hat{u} \\
\lambda-\hat{\lambda} \\
\alpha-\hat{\alpha} \\
\beta-\hat{\beta}
\end{array}\right)-\left(\begin{array}{c}
G(u, \hat{u}, \lambda, \hat{\lambda}) \\
0 \\
0 \\
0
\end{array}\right)
$$

where $G(u, \hat{u}, \lambda, \hat{\lambda})=(u-\hat{u})^{2}+(\lambda-\hat{\lambda})(u-\hat{u})$. This implies that

$$
\begin{gathered}
\left\|F_{0}(u, \lambda, \alpha, \beta)-F_{0}(\hat{u}, \hat{\lambda}, \hat{\alpha}, \hat{\beta})-D F_{0}(\hat{u}, \hat{\lambda}, \hat{\alpha}, \hat{\beta})\left(\begin{array}{c}
u-\hat{u} \\
\lambda-\hat{\lambda} \\
\alpha-\hat{\alpha} \\
\beta-\hat{\beta}
\end{array}\right)\right\|_{0} \\
\leq\|(\lambda-\hat{\lambda})(u-\hat{u})\|_{0}+\left\|(u-\hat{u})^{2}\right\|_{0} \\
\leq 2 \pi|\lambda-\hat{\lambda}|\|u-\hat{u}\|_{1}+8 \pi^{3}\|u-\hat{u}\|_{1}^{2} .
\end{gathered}
$$

Hence $F_{0}$ is continuously differentiable.

(ii) $D F_{0}(\bar{u}(\gamma), \lambda(\gamma), \alpha(\gamma), 0)$ is injective and surjective. We prove this property in Lemma A.5 in the appendix. 
(iii) $D F_{0}(\bar{u}(\gamma), \lambda(\gamma), \alpha(\gamma), 0)^{-1}\left[F(u, \lambda, \alpha, \beta ; \varepsilon)-F_{0}(u, \lambda, \alpha, \beta)\right]$ is Lipschitz continuous. This last property follows from the facts that $F$ and $F_{0}$ are Lipschitz continuous and $D F_{0}(\bar{u}(\gamma), \lambda(\gamma), \alpha(\gamma), 0)^{-1}$ is bounded. This last observation is a consequence of the minimality of the cnoidal waves and hence of Lemma 2.2 .

With (i)-(iii), all conditions for the application of the implicit-function theorem to the equation $F=0$ are satisfied.

2. If $\left.\left(R(\bar{u}, \varepsilon), \bar{u}_{x}\right)\right)=0$, then we consider the equations (19) on a subspace of $H_{\text {per }}^{1}$, namely

$$
\mathbb{X}(\gamma)=\left\{u \in H_{\text {per }}^{1} \mid\left(u, X_{I}(\bar{u}(\gamma))\right)=0\right\} .
$$

Also, in a way similar to case 1, we can prove that all conditions for the application of the implicit-function theorem to equation (19) are satisfied.

The application of the implicit-function theorem implies that there exists a neighborhood $\mathcal{U}_{1}(\gamma) \subset H_{\text {per }}^{1} \times \mathbb{R} \times \mathbb{R}$ around the relative equilibrium $(\bar{u}(\gamma), \lambda(\gamma), \alpha(\gamma))$, a positive number $\varepsilon_{0}(\gamma)$, and a curve of points $\left(\left(\bar{u}_{1}(\gamma, \varepsilon), \lambda_{1}(\gamma, \varepsilon), \alpha_{1}(\gamma, \varepsilon)\right)\right)_{|\varepsilon| \leq \varepsilon_{0}(\gamma)}$ in $\mathcal{U}_{1}(\gamma)$ such that

$$
\begin{aligned}
& 0=H^{\prime}\left(\bar{u}_{1}\right)-\lambda_{1} I^{\prime}\left(\bar{u}_{1}\right)-\alpha_{1}-\partial_{x}^{-1} R(\bar{u}, \varepsilon), \\
& 0=I\left(\bar{u}_{1}\right)-\gamma \\
& 0=M\left(\bar{u}_{1}\right) .
\end{aligned}
$$

Furthermore, it follows also that

$$
\begin{aligned}
\left\|\bar{u}_{1}(\gamma, \varepsilon)-\bar{u}(\gamma)\right\|_{1} & =\mathcal{O}\left(\left\|\partial_{x}^{-1} R(\bar{u}, \varepsilon)\right\|_{0}\right), \\
\left|\lambda_{1}(\gamma, \varepsilon)-\lambda(\gamma)\right| & =\mathcal{O}\left(\left\|\partial_{x}^{-1} R(\bar{u}, \varepsilon)\right\|_{0}\right), \\
\left|\alpha_{1}(\gamma, \varepsilon)-\alpha(\gamma)\right| & =\mathcal{O}\left(\left\|\partial_{x}^{-1} R(\bar{u}, \varepsilon)\right\|_{0}\right) .
\end{aligned}
$$

From the definition of the residual $R(\bar{u}, \varepsilon)$ (see (13)) and by using properties of elliptic functions (see [8]), it follows that there is a constant $K_{2}$ (independent of $\gamma$ ) such that $\|R(\bar{u}(\gamma), \varepsilon)\|_{0} \leq \varepsilon K_{2} \sqrt{ } \gamma$.

We must still show that $\bar{u}_{1}(\gamma, \varepsilon)$ is a constrained minimum on the level set with $I=\gamma$. Consider the linearization of $H^{\prime}(u)-\lambda_{1} I^{\prime}(u)-\partial_{x}^{-1} R(\bar{u}, \varepsilon)$ around $\bar{u}_{1}$ :

$$
\tilde{Q}_{1}(\gamma, \varepsilon)=D^{2} H\left(\bar{u}_{1}\right)-\lambda_{1} D^{2} I\left(\bar{u}_{1}\right)=Q(\gamma)-\left(\lambda_{1}-\lambda\right) I d-2\left(\bar{u}_{1}-\bar{u}\right) .
$$

Using the fact that $Q(\gamma)$ is strictly positive definite on $\mathbb{Y}(\gamma)$ and $(22)$, we will show that $\tilde{Q}_{1}(\gamma)$ is strictly positive definite on $\tilde{\mathbb{Y}}_{1}(\gamma)=\left\{\eta \in H_{\text {per }}^{1} \mid\left(\eta, X_{I}\left(\bar{u}_{1}\right)\right)=0,\left(\eta, I^{\prime}\left(\bar{u}_{1}\right)\right)=\right.$ $0\}$.

Let $\eta \in \tilde{\mathbb{Y}}_{1}(\gamma)$. Then we have

$$
\begin{aligned}
\left(\tilde{Q}_{1}(\gamma) \eta, \eta\right) & =(Q(\gamma) \eta, \eta)-\left(\lambda_{1}-\lambda_{0}\right)\|\eta\|_{0}^{2}-2\left(\left(\bar{u}_{1}-\bar{u}\right) \eta, \eta\right) \\
& \geq(Q(\gamma) \eta, \eta)-\left|\lambda_{1}-\lambda_{0}\right|\|\eta\|_{0}^{2}-2\left\|\bar{u}_{1}-\bar{u}\right\|_{1}\|\eta\|_{0}^{2} \\
& \geq(Q(\gamma) \eta, \eta)-3 K\left\|\partial_{x}^{-1} R_{3}(\bar{u}, \varepsilon)\right\|_{0}\|\eta\|_{0}^{2} .
\end{aligned}
$$

From the minimality of the cnoidal waves, it follows that there exists a $c(\gamma)$ such that $(Q(\gamma) \xi, \xi) \geq c(\gamma)\|\xi\|_{1}^{2}$ for all $\xi \in \mathbb{Y}(\gamma)$. We will use this to prove that $(Q(\gamma) \eta, \eta)$ is strictly positive. Write

$$
\eta=a X_{I}(\bar{u})+b I^{\prime}(\bar{u})+y
$$


with $y \in \mathbb{Y}(\gamma)$. Then we have

$$
\begin{aligned}
|a|=\left|\left(\eta, X_{I}(\bar{u})\right)\right| & =\left|0+\left(\eta, \partial_{x}\left(\bar{u}-\bar{u}_{1}\right)\right)\right| \leq K\left\|\partial_{x}^{-1} R_{3}(\bar{u}, \varepsilon)\right\|_{0}\|\eta\|_{0}, \\
|b|=\left|\left(\eta, I^{\prime}(\bar{u})\right)\right| & =\left|0+\left(\eta,\left(\bar{u}-\bar{u}_{1}\right)\right)\right| \leq K\left\|\partial_{x}^{-1} R_{3}(\bar{u}, \varepsilon)\right\|_{0}\|\eta\|_{0} .
\end{aligned}
$$

This implies that there exists some $\tilde{K}>0$ such that

$$
\begin{aligned}
(Q(\gamma) \eta, \eta) & =b^{2}(Q(\gamma) \bar{u}, \bar{u})+(Q(\gamma) y, y) \\
& \geq c(\gamma)\|y\|_{0}^{2}-K^{2}\left\|\partial_{x}^{-1} R_{3}(\bar{u}, \varepsilon)\right\|_{0}^{2}\|\eta\|_{0}^{2}\|Q(\gamma)\|_{0},\|\bar{u}\|_{0}^{2} \\
& \geq(c(\gamma)-\tilde{K} \varepsilon \sqrt{ } \gamma)\|\eta\|_{0}^{2} \\
& \geq \frac{1}{2} c(\gamma)\|\eta\|_{0}^{2}
\end{aligned}
$$

for $\varepsilon$ sufficiently small and $\gamma$ bounded. Hence

$$
\begin{aligned}
\left(\tilde{Q}_{1}(\gamma) \eta, \eta\right) & \geq \frac{1}{2} c(\gamma)\|\eta\|_{0}^{2}-3 K\left\|\partial_{x}^{-1} R_{3}(\bar{u}, \varepsilon)\right\|_{0}\|\eta\|_{0}^{2} \\
& \geq \frac{1}{4} c(\gamma)\|\eta\|_{0}^{2}
\end{aligned}
$$

for $\varepsilon$ sufficiently small. With Lemma A.4, this implies that

$$
\left(\tilde{Q}_{1}(\gamma) \eta, \eta\right) \geq{ }_{4}^{1} \tilde{c}_{1}(\gamma)\|\eta\|_{1}^{2}
$$

for some $\tilde{c}_{1}(\gamma)>0$ with $\tilde{c}_{1}(0)>0$. We can conclude that $\bar{u}_{1}(\gamma)$ is a constrained minimum of the new Hamiltonian $H_{\text {new }}$.

Finally, we consider the problem of the uniformness in $\gamma$. The procedure of the implicit-function theorem can be continued until the invertibility of the linearization fails. Because the lower bound on $\tilde{Q}(\gamma)$ is also strictly positive in the limit for $\gamma \rightarrow 0$, there is a uniform (in $\gamma$ ) neighborhood around the MRE near 0 for which a unique solution of (18) exists. In other words, the limit for $\gamma \rightarrow 0$ of $\varepsilon_{0}(\gamma)$ and $K(\gamma)$ exist and $\varepsilon_{0}(0)=0$.

Remark 6. At the new relative equilibrium $\bar{u}_{1}(\gamma, \varepsilon)$, the adapted residual is of or$\operatorname{der} \varepsilon^{2}\|\bar{u}(\gamma)\|_{0}$. Indeed,

$$
\begin{aligned}
R\left(\bar{u}_{1}(\gamma, \varepsilon), \varepsilon\right) & =\dot{\gamma} \bar{u}_{1}^{\prime}(\gamma, \varepsilon)-\partial H_{\text {new }}^{\prime}\left(\bar{u}_{1}, \gamma, \varepsilon\right)-R(\bar{u}, \varepsilon)-\varepsilon P\left(\bar{u}_{1}\right) \\
& =-2 \varepsilon \gamma\left[\bar{u}_{1}^{\prime}(\gamma, \varepsilon)-\bar{u}^{\prime}(\gamma)\right]-\varepsilon\left[\bar{u}_{1}-\bar{u}\right]-\lambda_{1} X_{I}\left(\bar{u}_{1}\right) .
\end{aligned}
$$

With $\left\|\bar{u}(\gamma)-\bar{u}_{1}(\gamma, \varepsilon)\right\|_{1}=\mathcal{O}\left(\varepsilon\|\bar{u}(\gamma)\|_{0}\right)$, we immediately see that $\left\|R\left(\bar{u}_{1}(\gamma, \varepsilon), \varepsilon\right)\right\|_{1}=$ $\mathcal{O}\left(\varepsilon^{2}\|\bar{u}(\gamma)\|_{0}\right)$.

With the new Hamiltonian and the new minima, we can define a functional to "measure" the distance to the new minima:

$$
L_{\text {new }}(u, \varepsilon)=H_{\text {new }}(u, \gamma, \varepsilon)-H_{\text {new }}\left(\bar{u}_{1}(\gamma, \varepsilon), \gamma, \varepsilon\right)
$$

with $\gamma=I(u)$. The functional $L_{\text {new }}$ is equivalent to the translation-invariant $H^{1}$ distance between $u$ and $\bar{u}_{1}(\gamma, \varepsilon)$ with $\gamma=I(u)$.

LEMma 3.2. For every $\gamma>0$, there exist $C(\gamma) \geq c(\gamma)>0, \delta(\gamma)>0$, and $\varepsilon_{0}(\gamma)>0$, such that for all $\varepsilon$ with $|\varepsilon| \leq \varepsilon_{0}(\gamma)$ and for all $\eta$ with $\left(\eta, X_{I}\left(\bar{u}_{1}+\eta\right)\right)=0$ and $I\left(\bar{u}_{1}(\gamma)+\eta\right)=\gamma$, it holds that

$$
c(\gamma)\|\eta\|_{1}^{2} \leq L_{\text {new }}\left(\bar{u}_{1}(\gamma, \varepsilon)+\eta, \varepsilon\right) \leq C(\gamma)\|\eta\|_{1}^{2}
$$


as long as $\|\eta\|_{1} \leq \delta(\gamma)$.

For every compact $\gamma$-interval $\mathcal{G}$, there exist $C \geq c>0, \delta>0$, and $\varepsilon_{0}>0$ such that for all $\varepsilon$ with $|\varepsilon| \leq \varepsilon_{0}$, for all $\gamma \in \mathcal{G}$, and for all $\eta$ with $\left(\eta, X_{I}\left(\bar{u}_{1}+\eta\right)\right)=0$ and $I\left(\bar{u}_{1}(\gamma)+\eta\right)=\gamma$, it holds that

$$
c\|\eta\|_{1}^{2} \leq L_{\text {new }}\left(\bar{u}_{1}(\gamma, \varepsilon)+\eta, \varepsilon\right) \leq C\|\eta\|_{1}^{2}
$$

as long as $\|\eta\|_{1} \leq \delta$.

Proof. In the proof of Lemma 3.1, it is shown that $\tilde{Q}_{1}(\gamma, \varepsilon)=D_{\eta}^{2} L_{\text {new }}\left(\bar{u}_{1}(\gamma, \varepsilon), \varepsilon\right)$ is bounded from below on $\tilde{\mathbb{Y}}(\gamma)=\left\{\eta \in L^{2} \mid\left(\eta, X_{I}\left(\bar{u}_{1}\right)\right)=0,\left(\eta, X_{I}\left(\bar{u}_{1}\right)\right)=0\right\}$ (see equation (23)) and that this lower bound remains strictly positive if $\gamma \rightarrow 0$. As in the proof of Lemma 2.2, we can show that (23) implies that there is some $c(\gamma)>0$ such that

$$
L_{\text {new }}\left(\bar{u}_{1}(\gamma, \varepsilon)+\eta, \varepsilon\right) \geq c(\gamma)\|\eta\|_{1}^{2}
$$

with $c(0)>0$.

For the upper bounds, we rewrite $L_{\text {new }}$ :

$$
\begin{aligned}
L_{\text {new }}\left(\bar{u}_{1}(\gamma, \varepsilon)+\eta, \varepsilon\right)= & H_{\text {new }}\left(\bar{u}_{1}(\gamma)+\eta, \gamma, \varepsilon\right)-\lambda_{1}(\gamma, \varepsilon) I\left(\bar{u}_{1}(\gamma)+\eta\right) \\
& -\left[H_{\text {new }}\left(\bar{u}_{1}(\gamma), \gamma, \varepsilon\right)-\lambda_{1}(\gamma, \varepsilon) I\left(\bar{u}_{1}(\gamma)\right)\right] \\
= & \left(H_{\text {new }}^{\prime}\left(\bar{u}_{1}(\gamma)\right)-\lambda_{1}(\gamma, \varepsilon) I^{\prime}\left(\bar{u}_{1}(\gamma)\right), \eta\right) \\
& +\frac{1}{2}\left(\tilde{Q}_{1}(\gamma, \varepsilon) \eta, \eta\right)-\frac{1}{3} \int_{0}^{2 \pi} \eta^{3}(x, t) d x .
\end{aligned}
$$

It is a straightforward calculation to derive the following estimates for $\|\eta\|_{1} \leq \delta$ :

$$
\begin{aligned}
& \left(Q_{1}(\gamma, \varepsilon) \eta, \eta\right) \leq\left\|\eta_{x}\right\|_{0}^{2}+\left(2\left\|\bar{u}_{1}\right\|_{\infty}+\left|\lambda_{1}\right|\right)\|\eta\|_{0}^{2} \leq C_{1}(\gamma)\|\eta\|_{1}^{2}, \\
& \int_{0}^{2 \pi} \eta^{3}(x, t) d x \leq\|\eta\|_{\infty}\|\eta\|_{0}^{2} \leq \sqrt{ } 2 \pi 4 \pi^{2} \delta\|\eta\|_{1}^{2} .
\end{aligned}
$$

Note that $C_{1}(\gamma)$ is bounded from above if $\gamma \rightarrow 0$ and that the second estimate in (24) does not depend on $\gamma$ at all. Substitution of the estimates in (24) gives the upper bounds in the lemma.

With $L_{\text {new }}$, we investigate the time behavior of the distance between a solution $u(t)$ of the damped KdV equation and the new relative equilibrium $\bar{u}_{1}(\gamma(t), \varepsilon)$. For a solution of the damped $\mathrm{KdV}$ equation, we define

$$
\eta(t)=\Phi_{\phi(t)}^{I}(u(t))-\bar{u}_{1}(\gamma(t), \varepsilon)
$$

or, equivalently,

$$
u(t)=\Phi_{\phi(t)}^{I}\left(\bar{u}_{1}(\gamma(t), \varepsilon)+\eta(t)\right)
$$

with $\phi(t)$ such that $\|\eta(t)\|^{2}=\rho_{1}\left(u(t), \bar{u}_{1}(\gamma(t)), \varepsilon\right)$ ). (This implies the property that $\left(\eta, X_{I}\left(\bar{u}_{1}+\eta\right)\right)=0$.) With this definition, the dynamical equation for $\eta$ is

$$
\dot{\eta}+\dot{\bar{u}}_{1}=\partial_{x} H_{\text {new }}^{\prime}\left(\bar{u}_{1}+\eta, \gamma, \varepsilon\right)-\dot{\phi} X_{I}\left(\bar{u}_{1}+\eta\right)+\left[\varepsilon P\left(\bar{u}_{1}+\eta\right)+R(\bar{u}, \varepsilon)\right] .
$$

Next, we give an estimate for the growth of $L_{\text {new }}$ which is essentially better than the one we derived for $L$ in the previous section. 
Proposition 3.3. For every $\gamma(0)$, there exists a constant $K$ (depending on $\gamma(0)$ ) such that for all $t \geq 0$ and $|\varepsilon| \leq \varepsilon_{0}$, it holds that

$$
L_{\text {new }}(u(t), \varepsilon) \leq K L_{\text {new }}(u(0), \varepsilon) e^{-2 \varepsilon t}+K \varepsilon e^{-2 \varepsilon t}
$$

as long as $\|\eta(t)\|_{1} \leq \delta$ ( $\delta$ is given by Lemma 3.2).

Proof. To prove this proposition, we consider the time derivative of $L_{\text {new }}$ :

$$
\begin{aligned}
\frac{\mathrm{d} f}{\mathrm{~d} t}\left[L_{\text {new }}(u(t), \varepsilon)\right]= & \left(H_{\text {new }}^{\prime}\left(\bar{u}_{1}+\eta, \gamma, \varepsilon\right)-\lambda_{1} I^{\prime}\left(\bar{u}_{1}+\eta\right), \dot{\bar{u}}_{1}+\dot{\eta}\right) \\
& \left.\left.+\dot{\gamma}\left[\frac{\partial}{\partial \gamma} H_{\text {new }}\left(\bar{u}_{1}+\eta, \gamma, \varepsilon\right)\right)-\frac{\partial}{\partial \gamma} H_{\text {new }}\left(\bar{u}_{1}, \gamma, \varepsilon\right)\right)\right] \\
= & (\mathrm{I})+(\mathrm{II}) .
\end{aligned}
$$

We will elaborate the terms (I) and (II) separately.

$$
\begin{aligned}
(\mathrm{I})= & -\varepsilon\left(H_{\text {new }}^{\prime}\left(\bar{u}_{1}+\eta, \gamma, \varepsilon\right)-\lambda_{1} I^{\prime}\left(\bar{u}_{1}+\eta\right), \bar{u}_{1}+\eta-\frac{1}{\varepsilon} R(\bar{u}, \varepsilon)\right) \\
= & -\varepsilon\left(\tilde{Q}_{1}(\gamma, \varepsilon) \eta-\eta^{2}, \bar{u}_{1}+\eta-\frac{1}{\varepsilon} R(\bar{u}, \varepsilon)\right) \\
= & -2 \varepsilon L_{\text {new }}+\frac{\varepsilon}{3} \int_{0}^{2 \pi} \eta^{3}+\left(\eta^{2},\left[\varepsilon \bar{u}_{1}-R(\bar{u}, \varepsilon)\right]\right) \\
& -\varepsilon\left(\eta, \tilde{Q}_{1}(\gamma, \varepsilon)\left[\bar{u}_{1}-\frac{1}{\varepsilon} R(\bar{u}, \varepsilon)\right]\right) .
\end{aligned}
$$

In the same way as we showed that $\|\xi(t)\|_{0} \leq 2 \sqrt{ } 2 \gamma(t)$ (see (8)), it can be seen that $\|\eta(t)\|_{0} \leq 2 \sqrt{ } 2 \gamma(t)$. Just as in the proof of Proposition 2.3, this implies that $\int_{0}^{2 \pi} \eta^{3} \leq 8 \pi \sqrt{ } \pi \sqrt{ } \gamma\|\eta\|_{1}^{2}=\mathcal{O}\left(\varepsilon^{2} \sqrt{ } \gamma+\varepsilon \sqrt{ } \gamma\right)$.

Furthermore, $\varepsilon \bar{u}_{1}-R(\bar{u}, \varepsilon)=\varepsilon\left(\bar{u}_{1}-\bar{u}\right)-\dot{\bar{u}}$; hence $\left\|\varepsilon \bar{u}_{1}-R(\bar{u}, \varepsilon)\right\|_{0} \leq \varepsilon \| \bar{u}_{1}-$ $\bar{u}\left\|_{0}+\right\| \dot{\bar{u}} \|_{0}$. In this estimate, we use the explicit expression for the cnoidal waves in terms of the Jacobi elliptic functions to conclude that $\|\dot{\bar{u}}\|_{0}=2 \varepsilon \gamma\left\|\bar{u}^{\prime}(\gamma)\right\|=\mathcal{O}(\varepsilon \sqrt{ } \gamma)$.

Using these estimates, we see that the second and third terms in (26) are bounded by $\varepsilon K_{4} \sqrt{ } \gamma\|\eta\|_{1}^{2}\left(K_{4}\right.$ is a constant independent of $\gamma$ and of $\varepsilon$ ). We will give more attention to the estimate of the last term because it will improve the estimate of Proposition 2.3. By definition,

$$
\varepsilon\left(\eta, \tilde{Q}_{1}(\gamma, \varepsilon)\left[\bar{u}_{1}-\frac{1}{\varepsilon} R(\bar{u}, \varepsilon)\right]\right)=\varepsilon\left(\eta, \tilde{Q}_{1}(\gamma, \varepsilon)\left[\left(\bar{u}_{1}-\bar{u}\right)-2 \gamma \bar{u}^{\prime}(\gamma)\right]\right) .
$$

Furthermore (see Lemma A.2(ii) in the appendix),

$$
\begin{aligned}
\tilde{Q}_{1}(\gamma, \varepsilon) \bar{u}^{\prime}(\gamma) & =Q(\gamma) \bar{u}^{\prime}(\gamma)+2\left(\bar{u}-\bar{u}_{1}\right) \bar{u}^{\prime}(\gamma) \\
& =\lambda^{\prime}(\gamma) \bar{u}_{1}+\left(\bar{u}-\bar{u}_{1}\right)\left[\lambda^{\prime}(\gamma)+2 \bar{u}^{\prime}(\gamma)\right] .
\end{aligned}
$$

Hence

$$
\begin{aligned}
\varepsilon\left(\eta, \tilde{Q}_{1}(\gamma, \varepsilon)\left[\bar{u}_{1}-\frac{1}{\varepsilon} R(\bar{u}, \varepsilon)\right]\right)= & \varepsilon\left(\eta,\left[\tilde{Q}_{1}(\gamma, \varepsilon)-2 \gamma\left(\lambda^{\prime}(\gamma)+2 \bar{u}^{\prime}(\gamma)\right)\right]\left(\bar{u}_{1}-\bar{u}\right)\right) \\
& -2 \varepsilon \gamma \lambda^{\prime}(\gamma)\left(\eta, \bar{u}_{1}\right) .
\end{aligned}
$$


By using the facts that $2\left(\eta, \bar{u}_{1}\right)=-\|\eta\|_{0}^{2}$ (this follows from $I\left(\bar{u}_{1}+\eta\right)=I\left(\bar{u}_{1}\right)$ ), $\gamma \lambda^{\prime}(\gamma)=\mathcal{O}(\sqrt{ } \gamma)$, and $\left\|\bar{u}(\gamma)-\bar{u}_{1}(\gamma, \varepsilon)\right\|_{1}=\mathcal{O}(\varepsilon \sqrt{ } \gamma)$ (see Lemma 3.1), it follows that there exist constants $K_{5}$ and $K_{6}$ such that

$$
\varepsilon\left(\eta, \tilde{Q}_{1}(\gamma)\left[\bar{u}_{1}-\frac{1}{\varepsilon} R(\bar{u}, \varepsilon)\right]\right) \leq \varepsilon^{2} K_{5} \sqrt{ } \gamma\|\eta\|_{1}+\varepsilon K_{6} \sqrt{ } \gamma\|\eta\|_{1}^{2} .
$$

Next, we estimate the second term of equation (25). Using the definitions of $H_{\text {new }}$ and $R(\bar{u}, \varepsilon)$, it follows that

$$
\begin{aligned}
(\mathrm{II}) & =\dot{\gamma}\left(\eta, \partial_{x}^{-1} \frac{\partial}{\partial \gamma}\left(2 \varepsilon \gamma \bar{u}^{\prime}(\gamma)+\varepsilon \bar{u}(\gamma)\right)\right. \\
& =-2 \varepsilon^{2} \gamma\|\eta\|_{0}\left\|\partial_{x}^{-1}\left(3 \bar{u}^{\prime}(\gamma)+2 \gamma \bar{u}^{\prime \prime}(\gamma)\right)\right\|_{0} \\
& \leq K_{7} \varepsilon^{2}\|\eta\|_{1} \sqrt{ } \gamma .
\end{aligned}
$$

(Again, we use the explicit expression for $\bar{u}$ in terms of the Jacobi elliptic functions.)

Finally, using the fact that $\|\eta\|^{2} \leq L_{\text {new }} / c$ (for $\|\eta\|_{1} \leq \delta$ ), we can estimate $\frac{\mathrm{d}}{\mathrm{d} t} L_{\text {new }}$ by

$$
\frac{\mathrm{d}}{\mathrm{d} t} L_{\text {new }} \leq-2 \varepsilon L_{\text {new }}+K_{0} \varepsilon \sqrt{ } \gamma L_{\text {new }}+K_{0} \varepsilon^{2} \gamma \sqrt{ } L_{\text {new }}
$$

for some constant $K_{0}$. Integrating this equation and applying Gronwall's lemma, we have that there exists some constant $K$ such that

$$
L_{\text {new }}(u(t), \varepsilon) \leq K L_{\text {new }}(\gamma(0), \eta(0), \varepsilon) e^{-2 \varepsilon t}+K \varepsilon e^{-2 \varepsilon t} .
$$

The proof of Theorem 1.2 is a corollary of Proposition 3.3.

Proof of Theorem 1.2. From Lemmas 3.2 and 3.3 and the fact that $\|\eta(t)\|^{2}=$ $\left.\rho_{1}\left(u(t), \bar{u}_{1}(\gamma(t)), \varepsilon\right)\right)$, it follows that

$$
\left.\left.\rho_{1}\left(u(t), \bar{u}_{1}(\gamma(t)), \varepsilon\right)\right) \leq K \rho_{1}\left(u(0), \bar{u}_{1}(\gamma(0)), \varepsilon\right)\right) e^{-\varepsilon t}+K \varepsilon e^{-\varepsilon t}
$$

if $\|\eta(0)\|_{1}$ is sufficiently small.

Now we use the fact that $\left\|\bar{u}_{1}(\gamma, \varepsilon)-\bar{u}(\gamma)\right\|_{1} \leq \hat{K} \varepsilon\|\bar{u}\|_{0}$ for some constant $\hat{K}$ (see Lemma 3.1), which yields

$$
\begin{aligned}
\rho_{1}(u(t), \bar{u}(\gamma(t))) \leq & \left.\rho_{1}\left(u(t), \bar{u}_{1}(\gamma(t)), \varepsilon\right)\right)+\left\|\bar{u}_{1}(\gamma(t), \varepsilon)-\bar{u}(\gamma(t))\right\|_{1} \\
\leq & \left.K \rho_{1}\left(u(0), \bar{u}_{1}(\gamma(0)), \varepsilon\right)\right) e^{-\varepsilon t}+\tilde{K} \varepsilon e^{-\varepsilon t}(1+\sqrt{ } 2 \gamma(0)) \\
\leq & K \rho_{1}(u(0), \bar{u}(\gamma(0))) e^{-\varepsilon t}+\tilde{K} \varepsilon e^{-\varepsilon t}(1+\sqrt{ } 2 \gamma(0)) \\
& +K\left\|\bar{u}_{1}(\gamma(0), \varepsilon)-\bar{u}(\gamma(0))\right\|_{1} e^{-\varepsilon t} \\
\leq & K \rho_{1}(u(0), \bar{u}(\gamma(0))) e^{-\varepsilon t}+\bar{K} \varepsilon e^{-\varepsilon t}
\end{aligned}
$$

for some constant $\bar{K}$. This completes the proof of Theorem 1.2.

Appendix. Some properties of the unperturbed KdV equation and cnoidal waves. As indicated in [4], the cnoidal waves are constrained minima of the Hamiltonian on level sets of $I$.

Lemma A.1. Let $\gamma>0$. For all $2 \pi$-periodic functions $u$ with mean value zero that satisfy $I(u)=\gamma$, it holds that

$$
H(u) \geq H(\bar{u}(\gamma))
$$


If $H(u)=H(\bar{u}(\gamma))$ and $I(u)=\gamma$, then $u$ is a cnoidal wave with $2 \pi$ as a minimal period. Explicitly, we have

$$
(I(u)=\gamma \wedge H(u)=H(\bar{u}(\gamma))) \Rightarrow \exists_{\phi \in \mathbb{R}}\left[u=\Phi_{\phi}^{I}(\bar{u}(\gamma))\right] .
$$

Furthermore, all cnoidal waves $\bar{u}(\gamma)$ satisfy the Euler-Lagrange equation

$$
\partial_{x}\left(H^{\prime}(u)-\lambda I^{\prime}(u)\right)=\partial_{x}\left(\bar{u}_{x x}+\bar{u}^{2}+\lambda \bar{u}\right)=0,
$$

and the cnoidal wave $\bar{u}(\gamma)$ is an unconstrained minimum of the modified KdV Hamiltonian $H_{\gamma}(u)=H(u)-\lambda(\gamma) I(u)$.

Proof. Let $\gamma>0$. We start by proving that the minimum of $H$ on the level set $I=\gamma$ exists. For this proof, we show that $H$ is a weakly lower semicontinuous (w.l.s.c.) functional that is coercive on the level set $I=\gamma$.

(i) First, we show coerciveness. For all $u \in H_{\text {per }, 0}^{1}$ with $I(u)=\gamma$, it holds that

$$
\left|\int_{0}^{2 \pi} u^{3}(x) d x\right| \leq\|u\|_{\infty} \int_{0}^{2 \pi} u^{2}(x) d x \leq \sqrt{ } 2 \pi\|u\|_{1} 2 \gamma
$$

(we used the Poincaré inequalities (4) and (5)). This gives

$$
H(u) \geq \frac{1}{2} \int_{0}^{2 \pi} u_{x}^{2}(x) d x-\frac{1}{3}\left|\int_{0}^{2 \pi} u^{3}(x) d x\right| \geq\|u\|_{1}\left[\begin{array}{c}
1 \\
2
\end{array}\|u\|_{1}-\frac{\sqrt{ } 2 \pi}{3} \gamma\right] .
$$

The last expression grows to infinity for $\|u\|_{1} \rightarrow \infty$.

(ii) Next, we prove weak lower semicontinuity. The norm is a w.l.s.c. functional; hence $\int_{0}^{2 \pi} u_{x}^{2}(x) d x=\|u\|_{1}^{2}$ is w.l.s.c. The term $\int_{0}^{2 \pi} u^{3}(x) d x$ is a functional that is even weakly continuous. To prove this, we use the fact that $H_{\mathrm{per}, 0}^{1}$ is embedded in $C^{0}$ and the embedding operator is strongly continuous. (See [30, p. 82].) Hence if the sequence $\left(u_{n}\right)_{n \in \mathbb{N}}$ converges weakly to $u$ in $H_{\mathrm{per}, 0}^{1}$, then this sequence is uniformly convergent to $u$. This implies that

$$
\lim _{n \rightarrow \infty} \int_{0}^{2 \pi} u_{n}^{3}(x) d x=\int_{0}^{2 \pi} u^{3}(x) d x
$$

which shows that the functional $\int_{0}^{2 \pi} u^{3}(x) d x$ is weakly continuous. In the same way, it is proved that the set $\left\{u \in H_{\mathrm{per}, 0}^{1} \mid \int_{0}^{2 \pi} u^{2}(x) d x=2 \gamma\right\}$ is weakly closed.

A coercive w.l.s.c. functional defined on a (sequentially) weakly closed set attains its infimum on this set. (See $[6, \S 6.1]$ and [31, Chap. 38].) This completes the proof that $H$ has a minimum on the level set with $I=\gamma$.

From variational calculus, it follows that this minimum satisfies the Euler-Lagrange equation

$$
H^{\prime}(u)-\lambda I^{\prime}(u)=\alpha
$$

for some Lagrange multipliers $\lambda$ and $\alpha$. Using properties of elliptic functions (see, e.g., [8] or [10]), it follows that the cnoidal waves with minimal period are unique solutions of such a equation with minimal value of $H$.

In several places, we use properties of the operators $Q(\gamma)$ and $\tilde{Q}(\gamma)$ and the cnoidal waves. We list some important ones.

Lemma A.2. The operators $Q(\gamma)$ and $\tilde{Q}(\gamma)$ and the cnoidal waves satisfy the following properties: 
(i) $Q(\gamma) \bar{u}_{x}(\gamma)=\tilde{Q}(\gamma) \bar{u}_{x}(\gamma)=0$;

(ii) $Q(\gamma) \bar{u}^{\prime}(\gamma)=\tilde{Q}(\gamma) \bar{u}^{\prime}(\gamma)=\lambda^{\prime}(\gamma) \bar{u}(\gamma)+\alpha^{\prime}(\gamma) \mathbf{1}$ and $\alpha^{\prime}(\gamma)=\frac{1}{\pi}$;

(iii) $\tilde{Q}(\gamma) \mathbf{1}=-2 \bar{u}-\lambda(\gamma) \mathbf{1}$;

(iv) $\lambda^{\prime}(\gamma)<0$;

(v) range $[\tilde{Q}(\gamma)]=\left\{u \in H_{\mathrm{per}}^{1} \mid\left(X_{I}(\bar{u}), u\right)=0\right\}$.

Proof. (i) The translation invariance of both $H$ and $I$ implies that $\tilde{Q}(\gamma) X_{I}(\bar{u})=0$; hence $\tilde{Q}(\gamma) \bar{u}_{x}(\gamma)=0$. Also, because $\bar{u}_{x} \in H_{\text {per }, 0}^{1}, Q(\gamma) \bar{u}_{x}(\gamma)=0$.

(ii) Differentiation of the Euler-Lagrange equation for the cnoidal waves, i.e.,

$$
-\bar{u}_{x x}(\gamma)-\bar{u}^{2}-\lambda(\gamma) \bar{u}(\gamma)-\alpha(\gamma)=0,
$$

with respect to $\gamma$ shows statement (ii) of the lemma. Integration of this EulerLagrange equation yields $\alpha(\gamma)={ }_{\pi}^{1} \gamma$ and hence $\alpha^{\prime}(\gamma)={ }_{\pi}^{1}$.

(iii) The equation follows immediately from the definition of $\tilde{Q}(\gamma)$.

(iv) The proof of this property can be found in [11].

(v) $\tilde{Q}(\gamma)$ is a self-adjoint operator, and in Lemma A.3 it is proved that $X_{I}(\bar{u})$ is the only eigenvector with eigenvalue 0 .

The cnoidal waves are minima of the modified KdV Hamiltonian $H_{\gamma}$. This implies that $Q(\gamma)$ is positive definite on $\mathbb{Y}(\gamma)$. In Lemma A.3, we show that a slightly stronger property holds.

Lemma A.3. The operator $Q(\gamma)$ is strictly positive definite on $\mathbb{Y}(\gamma)$. To be explicit, there is some $c_{1}(\gamma)>0$ such that

$$
(Q(\gamma) y, y) \geq c_{1}(\gamma)\|y\|_{1}^{2} \quad \text { for all } y \in \mathbb{Y}(\gamma)
$$

and $\lim _{\gamma \rightarrow 0} c_{1}(\gamma)>0$.

Proof. To prove this boundedness from below, we consider the eigenvalues of $Q(\gamma)$. These eigenvalues form a monotonically nondecreasing sequence in $\mathbb{R}$ :

$$
\lambda_{0} \leq \lambda_{1} \leq \lambda_{2} \leq \cdots \quad \text { with } \lim _{n \rightarrow \infty} \lambda_{n}=\infty .
$$

For the smallest eigenvalue $\lambda_{0}$, it holds that

$$
\lambda_{0}=\min \left\{(Q(\gamma) \xi, \xi) \mid \xi \in H_{\mathrm{per}, 0}^{1}, \int_{0}^{2 \pi} \xi^{2}=1\right\} .
$$

This number is negative because

$$
(Q(\gamma) \bar{u}(\gamma), \bar{u}(\gamma))=-\int_{0}^{2 \pi} \bar{u}(\gamma)^{3}<0 .
$$

The last inequality is based on the fact that $I(\bar{u})=I(-\bar{u})$ and $H(\bar{u})<H(-\bar{u})$ because of the minimality of $H(\bar{u})$ on the level set with $I=\gamma$. This implies that $-{ }_{3}^{1} \int_{0}^{2 \pi} \bar{u}^{3}<\frac{1}{3} \int_{0}^{2 \pi} \bar{u}^{3}$; in other words, $-{ }_{3}^{2} \int_{0}^{2 \pi} \bar{u}^{3}<0$.

The translation invariance of $H$ and $I$ implies that $Q(\gamma) \bar{u}_{x}=0$; hence $Q(\gamma)$ has at least one eigenvalue which equals zero for all $\gamma>0$.

At $\gamma=0$, the operator $Q(0)$ is equal to $-\left(D_{x x}+I d\right)$. The eigenvalues of this operator on $H_{\mathrm{per}, 0}^{1}$ are $\left(k^{2}-1\right), k \in \mathbb{N}$. All these eigenvalues are double. The continuity of $Q(\gamma)$ in $\gamma$ implies that for $\gamma>0$ in a neighborhood of 0 , it holds that

$$
\lambda_{0}(\gamma)<0, \quad \lambda_{1}(\gamma)=0, \quad \lambda_{2}(\gamma)>0 .
$$


$\lambda$

3

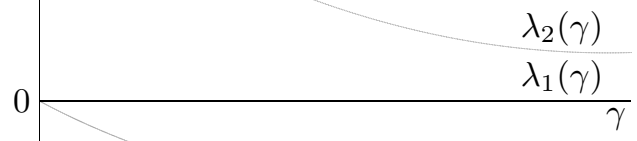

$\lambda_{0}(\gamma)$

FIG. 4. Sketch of the behavior of the eigenvalues $\lambda_{0}(\gamma), \lambda_{1}(\gamma)$, and $\lambda_{2}(\gamma)$.

See also Figure 4 .

We prove $\lambda_{2}(\gamma)>0$ for all $\gamma \geq 0$ by using a contradiction argument and $\tilde{Q}(\gamma)$, the extension of $Q(\gamma)$ on $H_{\mathrm{per}}^{1}$. Assume that there is some $\gamma_{0}>0$ such that $\lambda_{2}\left(\gamma_{0}\right)=0$. Then the operator $\tilde{Q}(\gamma)$ has a double eigenvalue zero. The differential equation for the eigenvalues and $2 \pi$-periodic eigenvectors of $\tilde{Q}(\gamma)$ is called Lamé's equation; see, e.g., [24]. It follows from Sturm-Liouville theory that zero is the second or the third eigenvalue of this equation because $\bar{u}_{x}$ is an eigenvector at zero. In [2], it is proved that the first three eigenvalues of this equation are single. Hence zero has to be a single eigenvalue of $\tilde{Q}(\gamma)$. This contradicts our assumption that $\lambda_{2}\left(\gamma_{0}\right)=0$ and implies that $\lambda_{2}\left(\gamma_{0}\right)>0$ for all $\gamma_{0} \geq 0$. We have seen that $\lambda_{2}(0)=3$; hence on every compact $\gamma$-interval, there is a positive lower bound for $\lambda_{2}(\gamma)$.

Using ideas similar to those of [23], this behavior of the eigenvalues of $Q(\gamma)$ implies that there is a $c_{0}(\gamma)>0$ such that

$$
\int_{0}^{2 \pi}\left[y_{x}^{2}(x)-(2 \bar{u}(x)-\lambda) y^{2}(x)\right] d x=(Q(\gamma) y, y) \geq c_{0}(\gamma)\|y\|_{0}^{2}
$$

for all $y \in \mathbb{Y}(\gamma)$. In Lemma A.4, we will prove that (37) implies that

$$
(Q(\gamma) y, y) \geq c_{1}(\gamma)\|y\|_{1}^{2} \quad \text { for all } y \in \mathbb{Y}(\gamma)
$$

with $c_{1}(\gamma)=\frac{c_{0}(\gamma)}{c_{0}(\gamma)+\|2 \bar{u}(\gamma)+\lambda(\gamma)\|_{\infty}}$.

In the proof of Lemma A.3, we used the fact that if $(Q(\gamma) y, y)$ is bounded from below in the $L^{2}$-norm for all $y \in \mathbb{Y}(\gamma)$, then it is bounded from below in the $H^{1}$-norm as well. This property can be concluded immediately from the following lemma.

Lemma A.4. Let $p(x)$ be a continuous function on $[-\pi, \pi]$. If for some $\xi \in H_{\mathrm{per}, 0}^{1}$ it holds that

$$
\int_{0}^{2 \pi}\left[\xi_{x}^{2}(x)+p(x) \xi^{2}(x)\right] d x \geq c\|\xi\|_{0}^{2}
$$

then

$$
\int_{0}^{2 \pi}\left[\xi_{x}^{2}(x)+p(x) \xi^{2}(x)\right] d x \geq c_{1}\|\xi\|_{1}^{2}
$$

with $c_{1}=\frac{c}{c+\|p\|_{\infty}}$. 
Proof. Assume that (38) holds for some $\xi \in H_{\text {per }, 0}^{1}$. By rewriting the integral in equation (38), we see that

$$
\begin{aligned}
& \int_{0}^{2 \pi}\left[\xi_{x}^{2}(x)+p(x) \xi^{2}(x)\right] d x=c_{1} \int_{0}^{2 \pi} \xi_{x}^{2}(x) d x+\left(1-c_{1}\right) \int_{0}^{2 \pi} \xi_{x}^{2}(x) d x \\
&+\int_{0}^{2 \pi} p(x) \xi^{2}(x) d x .
\end{aligned}
$$

Now we use inequality (38); it follows that

$$
\begin{aligned}
\int_{0}^{2 \pi}\left[\xi_{x}^{2}(x)+p(x) \xi^{2}(x)\right] d x \\
\quad \geq c_{1} \int_{0}^{2 \pi} \xi_{x}^{2}(x) d x+\left(1-c_{1}\right) c\|\xi\|_{0}^{2}-c_{1}\|p\|_{\infty}\|\xi\|_{0}^{2} \\
\quad=c_{1} \int_{0}^{2 \pi} \xi_{x}^{2}(x) d x+c\|\xi\|_{0}^{2}-c_{1}\left(c+\|p\|_{\infty}\right)\|\xi\|_{0}^{2} \\
\quad=c_{1}\|\xi\|_{1}^{2} .
\end{aligned}
$$

The last subject in this appendix is the operator $D F_{0}(\bar{u}(\gamma), \lambda(\gamma), \alpha(\gamma), 0)$ as defined in the proof of Lemma 3.1.

Lemma A.5. The operator $D F_{0}(\bar{u}(\gamma), \lambda(\gamma), \alpha(\gamma), 0)$ is injective and surjective, for all $\gamma>0$.

Proof. Let $\gamma>0$. Define $A_{0}$ on $H_{\text {per }}^{1} \times \mathbb{R} \times \mathbb{R} \times \mathbb{R}$ as

$$
A_{0}=D F_{0}(\bar{u}(\gamma), \lambda(\gamma), \alpha(\gamma), 0)=\left(\begin{array}{cccc}
\tilde{Q}(\gamma) & -\bar{u}(\gamma) & -\mathbf{1} & -\bar{u}_{x}(\gamma) \\
\bar{u}(\gamma) & 0 & 0 & 0 \\
\mathbf{1} & 0 & 0 & 0 \\
R(\bar{u}(\gamma), \varepsilon) & 0 & 0 & 0
\end{array}\right)
$$

(i) First, we prove that $A_{0}$ is an injective map. Assume that $A_{0}(v, l, a, b)=0$ for some $(v, l, a, b) \in H_{\text {per }}^{1} \times \mathbb{R} \times \mathbb{R} \times \mathbb{R}$; hence

$$
\begin{aligned}
& 0=\tilde{Q}(\gamma) v-l \bar{u}(\gamma)-a \mathbf{1}-b \bar{u}_{x}(\gamma) \\
& 0=(\bar{u}(\gamma), v) \\
& 0=(\mathbf{1}, v) \\
& 0=(R(\bar{u}(\gamma), \varepsilon), v)
\end{aligned}
$$

Taking the inner product of (42) with $\bar{u}_{x}$ yields $b\left\|\bar{u}_{x}\right\|_{0}^{2}=0$ and hence $b=0$. Write $v=$ $c_{1} \bar{u}^{\prime}(\gamma)+c_{2} \bar{u}_{x}+y$, with $y \in \tilde{\mathbb{Y}}(\gamma)$. This decomposition is unique because $\left(\bar{u}^{\prime}(\gamma), \bar{u}\right)=$ ${ }_{2}^{1} \mathrm{~d} \gamma\|\bar{u}\|_{0}^{2}=1$ and hence $\bar{u}^{\prime}(\gamma) \notin \tilde{\mathbb{Y}}(\gamma)$. From $(43)$, it follows that $c_{1}=(v, \bar{u})=0$. Taking the inner product of (42) with $v$ shows that $0=(\tilde{Q}(\gamma) v, v)=(\tilde{Q}(\gamma) y, y)$. Hence $y=0$ because $\tilde{Q}(\gamma)$ is strictly positive definite on $\tilde{Y}(\gamma)$. From (45), it follows that $0=c_{2}\left(R(\bar{u}(\gamma), \varepsilon), \bar{u}_{x}\right)$. Because of the assumption that $\left(R(\bar{u}(\gamma), \varepsilon), \bar{u}_{x}\right) \neq 0$, this implies that $c_{2}=0$ and hence $v=0$. Finally, substituting $v=0$ and $b=0$ in (42) yields $l \bar{u}(\gamma)+a \mathbf{1}=0$. Because $\bar{u}(\gamma)$ and $\mathbf{1}$ are linearly independent, this implies that $l=0$ and $a=0$. 
(ii) Next, we show that $A_{0}$ is surjective. Assume that $A_{0}(v, l, a, b)=(w, m, c, d)$ for some $(v, l, a, b),(w, m, c, d) \in H_{\mathrm{per}}^{1} \times \mathbb{R} \times \mathbb{R} \times \mathbb{R}$; hence

$$
\begin{aligned}
w & =\tilde{Q}(\gamma) v-l \bar{u}(\gamma)-a \mathbf{1}-b \bar{u}_{x}(\gamma), \\
m & =(\bar{u}(\gamma), v), \\
c & =(\mathbf{1}, v), \\
d & =(R(\bar{u}(\gamma), \varepsilon), v) .
\end{aligned}
$$

Taking the inner product of (46) with $\bar{u}_{x}$ yields $b\left\|\bar{u}_{x}\right\|_{0}^{2}=\left(w, \bar{u}_{x}\right)$. This defines $b$ because $\left\|\bar{u}_{x}\right\|_{0}^{2} \neq 0$. Take the inner product of (46) with 1 and use Lemma A.2(iii), which yields $2 \pi a=-(w, \mathbf{1})-2 m-\lambda c$. This defines $a$. Take the inner product of (46) with $\bar{u}^{\prime}(\gamma)$ and use Lemma A.2(ii), which yields $l=-\left(w, \bar{u}^{\prime}(\gamma)\right)-b\left(\bar{u}_{x}, \bar{u}^{\prime}(\gamma)\right)+$ $\lambda^{\prime}(\gamma) m+\alpha^{\prime}(\gamma) c$. This defines $l$. From Lemma A.2(v), it follows that $\tilde{Q}(\gamma)$ is invertible on $\left\{\bar{u}_{x}\right\}^{\perp}$; hence

$$
v=\tilde{Q}(\gamma)^{-1}\left[w+l \bar{u}+a \mathbf{1}+b \bar{u}_{x}\right]+f \bar{u}_{x} .
$$

The value of $f$ follows from (49).

\section{REFERENCES}

[1] V. I. Arnold, Dynamical Systems III, Encyclopaedia of Mathematical Sciences, vol. 3, Springer-Verlag, Berlin, Heidelberg, 1988.

[2] F. M. Arscott, Periodic Differential Equations, Monographs in Pure and Applied Mathematics, vol. 66, Pergamon Press, Oxford, 1964.

[3] T. B. Benjamin, The stability of solitary waves, Proc. Roy. Soc. London Ser. A, 328 (1972), pp. 153-183.

[4] , Lectures on nonlinear wave motion, in Lectures in Applied Mathematics, vol. 15, American Mathematical Society, Providence, RI, 1974, pp. 3-47.

[5] T. B. Benjamin, J. L. Bona, And J. J. Mahony, Model equations for long waves in nonlinear dispersive systems, Philos. Trans. Roy. Soc. London Ser. A, 272 (1972), pp. 47-78.

[6] M. Berger, Nonlinearity and Functional Analysis, Pure and Applied Mathematics, vol. 74, Academic Press, New York, 1977.

[7] J. L. Bona And R. Smith, The initial value problem for the Korteweg-de Vries equation, Philos. Trans. Roy. Soc. London Ser. A, 278 (1975), pp. 555-604.

[8] P. F. Byrd and M. D. Friedman, Handbook of Elliptic Integrals for Engineers and Scientist, Grundlehren der mathematischen Wissenschaften 67, 2nd ed., Springer-Verlag, Berlin, 1971 (revised version).

[9] K. Deimling, Nonlinear Functional Analysis, Springer-Verlag, Berlin, 1985.

[10] G. DeRKs, Coherent structures in the dynamics of perturbed Hamiltonian systems, Ph.D. thesis, University of Twente, Twente, The Netherlands, 1992.

[11] G. Derks And S. A. VAn Gils, On the uniqueness of traveling waves in perturbed Korteweg-de Vries equations, Japan J. Indust. Appl. Math., 10 (1993), pp. 413-430.

[12] G. Derks, D. Lewis, And T. Ratiu, Approximations with curves of relative equilibria in Hamiltonian systems with dissipation, Nonlinearity, 8 (1995), pp. 1087-1113.

[13] G. Derks and T. P. Valkering, Approximation in a damped Hamiltonian system by successive relative equilibria, Japan J. Indust. Appl. Math., 9 (1992), pp. 141-161.

[14] J. M. Ghidaglia, Weakly damped forced Korteweg-de Vries equations behave like a finite dimensional dynamical system in the long time, J. Differential Equations, (1988), pp. 369390.

[15] M. Grillakis, J. Shatah, and W. Strauss, Stability theory of solitary waves in the presence of symmetry I, J. Funct. Anal., 74 (1987), pp. 160-197.

[16] E. van Groesen, F. P. H. van Beckum, and T. P. Valkering, Decay of travelling waves in dissipative Poisson systems, Z. Angew. Math. Phys., 41 (1990), pp. 501-523.

[17] D. D. Holm, J. E. Marsden, T. Ratiu, and A. Weinstein, Nonlinear stability of fluid and plasma equiliberia, Phys. Rep., 123 (1985), pp. 1-116.

[18] V. I. Karpman and E. M. Maslov, A perturbation theory for the Korteweg-de Vries equation, Phys. Lett., 60A (1977), pp. 307-308. 
[19] D. J. Kaup AND A. C. Newell, Solitons as particles, oscillators, and in slowly changing media: a singular perturbation theory, Proc. Roy. Soc. London Ser. A, 361 (1978), pp. 413-446.

[20] C. J. Knickerbocker And A. C. Newell, Shelves and the Korteweg-de Vries equation, J. Fluid Mech., 98 (1980), pp. 803-818.

[21] D. J. Korteweg And G. DEVRies, On the change of form of long waves advancing in a rectangular canal, and on a new type of long stationary waves, Philos. Magazine, 39 (1895), pp. $422-443$.

[22] N. R. Lebovitz and A. Neishtadt, Slow evolution in perturbed Hamiltonian systems, Stud. in Appl. Math., 92 (1994), pp. 127-144.

[23] J. H. Maddocks and R. L. Sachs, On the stability of KdV multi-solitons, Comm. Pure Appl. Math., 46 (1993), pp. 867-901.

[24] W. Magnus and S. Winkler, Hill's Equation, Interscience Tracts in Pure and Applied Mathematics, vol. 20, Interscience Publishers, New York, 1966.

[25] J. W. MiLEs, The Korteweg-de Vries equation: A historical essay, J. Fluid Mech., 106 (1981), pp. 131-147.

[26] P. J. Olver, Applications of Lie groups to differential equations, Springer-Verlag, New York, 1986.

[27] A. C. Scott, F. Y. F. Chu, and D. W. McLaughlin, The soliton: A new concept in applied science, Proc. IEEE, 61 (1973), pp. 1443-1483.

[28] J. C. Simo, D. Lewis, And J. E. Marsden, Stability of relative equilibria, part I: The reduced energy momentum method, Arch. Rational Mech. Anal., 115 (1991), pp. 15-60.

[29] J. C. Simo, T. A. Posbergh, and J. E. Marsden, Stability of relative equilibria, part II: Application to nonlinear elasticity, Arch. Rational Mech. Anal., 115 (1991), pp. 60-100.

[30] S. L. Sobolev, Applications of Functional Analysis in Mathematical Physics, Americal Mathematical Society, Providence, RI, 1963.

[31] E. ZeIdLeR, Nonlinear Functional Analysis and Its Applications, Part III: Variational Methods and Optimization, Springer-Verlag, Berlin, New York, Heidelberg, 1985. 